\title{
On a geometric equation involving the Sobolev trace critical exponent
}

\author{
Mohamed Ali Al-Ghamdi, Hichem Chtioui* and Khadija Sharaf
}

"Correspondence:

Hichem.Chtioui@fss.rnu.tn

Department of Mathematics, King

Abdulaziz University, Jeddah, Saudi

Arabia

\begin{abstract}
In this paper we consider the problem of prescribing the mean curvature on the boundary of the unit ball of $\mathbb{R}^{n}, n \geq 4$. Under the assumption that the prescribed function is flat near its critical point, we give precise estimates on the losses of the compactness, and we provide a new existence result of Bahri-Coron type. Moreover, we establish, under generic boundary condition, a Morse inequality at infinity, which gives a lower bound on the number of solutions to the above problem.
\end{abstract}

MSC: 58E05; 35J65; 53C21; 35B40

Keywords: boundary mean curvature; variational problem; loss of compactness; $\beta$-flatness condition; critical point at infinity

\section{Introduction}

In this paper we consider a nonlinear elliptic equation involving the Sobolev trace critical exponent associated to conformal deformations of Riemannian metrics on manifolds with boundary. We are interested in the case in which a non-compact group of conformal transformations acts on the equation giving rise to Kazdan-Warner type obstructions, just as in the celebrated scalar curvature problem (see [1]). The simplest situation is the following.

Let $B^{n}$ be the unit ball in $\mathbb{R}^{n}, n \geq 3$, with the Euclidean metric $g_{0}$. Its boundary is denoted by $S^{n-1}$ and it is endowed with the standard metric still denoted by $g_{0}$. Let $H: S^{n-1} \rightarrow \mathbb{R}$ be a given function. We study the problem of finding a conformal metric $g=u^{\frac{4}{n-2}} g_{0}$ such that $R_{g}=0$ in $B^{n}$ and $h_{g}=H$ on $S^{n-1}$. Here $R_{g}$ is the scalar curvature of the metric $g$ in $B^{n}$ and $h_{g}$ is the mean curvature of $g$ on $S^{n-1}$.

This problem has the following analytical formulation: find a smooth positive function which solves the following nonlinear boundary value equation:

$$
\left\{\begin{array}{l}
\Delta u=0 \quad \text { in } B^{n}, \\
\frac{\partial u}{\partial \nu}+\frac{n-2}{2} u=\frac{n-2}{2} H u^{\frac{n}{n-2}} \quad \text { on } S^{n-1},
\end{array}\right.
$$

where $v$ is the outward unit vector with respect to the metric $g_{0}$.

In general, there are several difficulties in facing this problem by means of variational methods. Indeed, by virtue of non-compactness of the embedding $H^{1}\left(B^{n}\right) \hookrightarrow L^{\frac{2(n-1)}{n-2}}\left(\partial B^{n}\right)$, the Euler-Lagrange functional $J$ associated to (1.1) does not satisfy the Palais-Smale condition, and that leads to the failure of the standard critical point theory. Moreover, besides the obvious necessary condition that $H$ must be positive somewhere, there is, as

(c) 2013 Al-Ghamdi et al.; licensee Springer. This is an Open Access article distributed under the terms of the Creative Commons Attribution License (http://creativecommons.org/licenses/by/2.0), which permits unrestricted use, distribution, and reproduction in any medium, provided the original work is properly cited. 
we have already mentioned, another obstruction to solving the problem, the so-called Kazdan-Warner obstruction. There have been many papers on the problem and related ones, please see [2-16] and the references therein.

One group of existence results has been obtained under hypotheses involving the Laplacian $\Delta H$ at the critical points $y$ of $H$; see $[17,18]$ for $n=3$, and [19-21] for $n \geq 4$. For example, in [17] and [18], it is assumed that $H$ is a Morse function and

$$
\Delta H(y) \neq 0 \quad \text { whenever } \quad \nabla H(y)=0 .
$$

Then, if $\operatorname{ind}(H, y)$ denotes the Morse index of $H$ at the critical point $y$, problem (1.1) has a solution provided

$$
\sum_{\Delta H(y)<0}(-1)^{\operatorname{ind}(H, y)} \neq 1
$$

The result has been extended to any dimension $n \geq 3$ in [19]. Roughly, it is assumed that there exists $\beta, n-2<\beta<n-1$, such that in some geodesic normal coordinate system centered at $y$, we have

$$
H(x)=H(0)+\sum_{i=1}^{n-1} b_{i}\left|\left(x_{i}\right)\right|^{\beta}+R(x),
$$

where $b_{i}=b_{i}(y) \in \mathbb{R} \backslash\{0\}, \forall i=1, \ldots, n-1, \sum_{i=1}^{n-1} b_{i} \neq 0$ and $\sum_{s=0}^{[\beta]}\left|\nabla^{s} R(x)\right||x|^{s-\beta}=o(1)$ as $x$ tends to zero. Here $\nabla^{s}$ denotes all possible derivatives of order $s$ and $[\beta]$ is an integer part of $\beta$. Let

$$
\mathcal{K}=\left\{y \in S^{n-1}, \nabla H(y)=0\right\}, \quad \mathcal{K}^{+}=\left\{y \in \mathcal{K}, \sum_{i=1}^{n-1} b_{i}<0\right\}
$$

and $\tilde{i}(y)=\sharp\left\{b_{i}, i=1, \ldots, n-1\right.$, such that $\left.b_{i}<0\right\}$. Then (1.1) has a solution provided

$$
\sum_{y \in \mathcal{K}^{+}}(-1)^{n-1-\tilde{i}(y)} \neq 1
$$

see [22].

Let us observe that a condition like (1.2) appeared first in [23] concerning the scalar curvature problem; see also [24].

In this work we restrict our attention to problem (1.1) under condition that $H$ is a $C^{2}$-function satisfying $(\mathfrak{f})_{\beta}$ condition with $n-2 \leq \beta<n-1$. This leads to an interesting new phenomenon, that is, the presence of multiple blow-up points. In fact, when looking to the possible formations of blow-up points, it comes out that the strong interaction of the bubbles in the case where $n-2<\beta<n-1$ forces all blow-up points to be single, while in the case where $\beta=n-2$, we have a balance phenomenon, that is, any interaction of two bubbles is of the same order with respect to the self-interaction. We denote by $\Xi$ the operator which associates to $H$ the solution $v$ of (1.1), and we extend the definition of $\Xi$ to the case of weak solutions of (1.1). Let

$$
\mathcal{K}_{n-2}=\{y \in \mathcal{K}, \beta=\beta(y)=n-2\}
$$


For every $\left(y_{i_{1}}, \ldots, y_{i_{N}}\right) \subseteq \mathcal{K}^{+} \cap \mathcal{K}_{n-2}$ such that $y_{i_{p}} \neq y_{i_{q}}$, if $p \neq q$, we associate the matrix $M=\left(M_{j l}\right)$ defined by

$$
\left\{\begin{array}{l}
M_{j j}=\frac{n-2}{n-1} \tilde{c}_{1} \frac{-\sum_{k=1}^{n-1} b_{k}\left(y_{i_{j}}\right)}{H\left(y_{l_{j}}\right)^{n-1}}, \quad j \in\{1, \ldots, N\} \\
M_{l j}=-2^{\frac{n-2}{2}} c_{1} \frac{G\left(y_{i}, y_{i_{j}}\right)}{\left[H\left(y_{i_{l}}\right) H\left(y_{i_{j}}\right)\right]^{\frac{n-2}{2}}}, \quad l, j \in\{1, \ldots, N\}, l \neq j,
\end{array}\right.
$$

where

$$
c_{1}=c_{0}^{\frac{2(n-1)}{n-2}} \int_{\mathbb{R}^{n-1}} \frac{d x}{\left(1+|x|^{2}\right)^{\frac{n}{2}}} \quad \text { and } \quad \tilde{c}_{1}=c_{0}^{\frac{2(n-1)}{n-2}} \int_{\mathbb{R}^{n-1}} \frac{\left|x_{1}\right|^{\beta}}{\left(1+|x|^{2}\right)^{n-1}} d x \text {. }
$$

Here $G(q, \cdot)$ denotes Green's function for the operator $\Xi$ with point $q$.

Let $\rho=\rho\left(y_{i_{1}}, \ldots, y_{i_{N}}\right)$ be the least eigenvalue of $M$. We assume the following:

$\left(A_{0}\right) \rho\left(y_{i_{1}}, \ldots, y_{i_{N}}\right) \neq 0$ for distinct points $y_{i_{1}}, \ldots, y_{i_{N}} \in \mathcal{K}^{+} \cap \mathcal{K}_{n-2}$.

We now introduce the following set:

$$
\begin{aligned}
C_{n-2}^{+}= & \left\{\left(y_{i_{1}}, \ldots, y_{i_{N}}\right), N \geq 1, \text { s.t. } y_{i_{j}} \in \mathcal{K}^{+} \cap \mathcal{K}_{n-2}\right. \\
& \left.\forall j=1, \ldots, N, y_{i_{j}} \neq y_{i_{l}} \forall j \neq l \text { and } \rho\left(y_{i_{1}}, \ldots, y_{i_{N}}\right)>0\right\} .
\end{aligned}
$$

We then have the following theorem.

Theorem 1.1 Assume that $H$ is a $C^{2}$-function satisfying $\left(A_{0}\right)$ and $(\mathfrak{f})_{\beta}$, with

$$
n-2 \leq \beta<n-1 \text {. }
$$

If

$$
\sum_{y \in \mathcal{K}^{+} \backslash \mathcal{K}_{n-2}}(-1)^{n-1-\tilde{i}(y)}+\sum_{\left(y_{i_{1}}, \ldots, y_{i_{N}}\right) \in \mathcal{C}_{n-2}^{+}}(-1)^{N-1+\sum_{j=1}^{N} n-1+\tilde{i}\left(y_{i j}\right)} \neq 1
$$

then (1.1) has at least one solution. Moreover, for generic $H$, we have

$$
\sharp S \geq\left|1-\sum_{y \in \mathcal{K}^{+} \backslash \mathcal{K}_{n-2}}(-1)^{n-1-\tilde{i}(y)}-\sum_{\left(y_{i_{1}}, \ldots, y_{i_{N}}\right) \in \mathcal{C}_{n-2}^{+}}(-1)^{N-1+\sum_{j=1}^{N} n-1+\tilde{i}\left(y_{i j}\right)}\right|,
$$

where $S$ denotes the set of solutions of (1.1).

Our argument uses a careful analysis of the lack of compactness of the Euler-Lagrange functional $J$ associated to problem (1.1). Namely we study the noncompact orbits of the gradient-flow of $J$, the so-called critical points at infinity following the terminology of Bahri [25]. These critical points at infinity can be treated as usual critical points once a Morse lemma at infinity is performed, from which we can derive, just as in the classical Morse theory, the difference of topology induced by these noncompact orbits and compute their Morse index. Such a Morse lemma at infinity is obtained through the construc- 
tion of a suitable pseudo-gradient, for which the Palais-Smale condition is satisfied along the decreasing flow lines as long as these flow lines do not enter the neighborhood of some specific critical points of $H$.

A similar Morse lemma at infinity has been established for problem (1.1) under the hypothesis that the function $H$ is of class $C^{2}$ and the order of flatness at critical points of $H$ is $\beta \in] n-2, n-1$ [; see [22].

The rest of this paper is organized as follows. In Section 2, we set up the variational problem and we recall the expansion of the gradient of the associated Euler-Lagrange functional near infinity. In Section 3, we construct a suitable pseudo-gradient and we characterize the critical points at infinity. Lastly, in Section 4, we prove our main result.

\section{General framework and some known facts}

\subsection{Variational problem}

First, we recall the functional setting and the variational problem and its main features. Problem (1.1) has a variational structure. The Euler-Lagrange functional is

$$
J(u)=\left(\int_{\mathbb{S}^{n-1}} H u^{\frac{2(n-1)}{n-2}} d \sigma_{g_{0}}\right)^{\frac{2-n}{n-1}},
$$

defined on $H^{1}\left(\mathbb{B}^{n}\right)$ equipped with the norm

$$
\|u\|^{2}=\int_{\mathbb{B}^{n}}|\nabla u|^{2} d v_{g_{0}}+\frac{n-2}{2} \int_{\mathbb{S}^{n-1}} u^{2} d \sigma_{g_{0}}
$$

where $d v_{g_{0}}$ and $d \sigma_{g_{0}}$ denote the Riemannian measure on $\mathbb{B}^{n}$ and $\mathbb{S}^{n-1}$ induced by the metric $g_{0}$. We denote by $\Sigma$ the unit sphere of $H^{1}\left(\mathbb{B}^{n}\right)$, and we set

$$
\Sigma^{+}=\{u \in \Sigma / u \geq 0\}
$$

The exponent $\frac{2(n-1)}{n-2}$ is critical for the Sobolev trace embedding $H^{1}\left(\mathbb{B}^{n}\right) \rightarrow L^{q}\left(\mathbb{S}^{n-1}\right)$. This embedding being not compact, the functional $J$ does not satisfy the Palais-Smale condition.

In order to characterize the sequences failing the Palais-Smale condition, we need to introduce some notations.

We use the notation $x$ for the variables belonging to the unit ball $\mathbb{B}^{n}$ or to the halfspace $\mathbb{R}_{+}^{n}$ defined by $\mathbb{R}_{+}^{n}:=\left\{x \in \mathbb{R}^{n}, x_{n}>0\right\}$. We also use the notation $x=\left(x^{\prime}, x_{n}\right)$ for $x \in \mathbb{R}_{+}^{n}$. It is convenient to perform some stereographic projection in order to reduce the above problem to $\mathbb{R}_{+}^{n}$. Let $D^{1,2}\left(\mathbb{R}_{+}^{n}\right)$ denote the completion of $C_{c}^{\infty}\left(\overline{\mathbb{R}}_{+}^{n}\right)$ with respect to the Dirichlet norm. The stereographic projection $\pi_{q}$ through an appropriate point $q \in \mathbb{S}^{n-1}$ induces an isometry $i: H^{1}\left(\mathbb{B}^{n}\right) \rightarrow D^{1,2}\left(\mathbb{R}_{+}^{n}\right)$ according to the following formula:

$$
i u(x)=\left(\frac{2}{\left|x^{\prime}\right|^{2}+\left(x_{n}+1\right)^{2}}\right)^{\frac{n-2}{2}} u\left(\frac{2 x^{\prime}}{\left|x^{\prime}\right|^{2}+\left(x_{n}+1\right)^{2}}, \frac{\left|x^{\prime}\right|^{2}+x_{n}-1}{\left|x^{\prime}\right|^{2}+\left(x_{n}+1\right)^{2}}\right),
$$


where $x^{\prime}=\left(x_{1}, \ldots, x_{n-1}\right)$. In particular, we can check that the following relations hold true for every $u \in H^{1}\left(\mathbb{B}^{n}\right)$ :

$$
\begin{aligned}
& \int_{\mathbb{B}^{n}}|\nabla u|^{2}+\frac{n-2}{2} \int_{\mathbb{S}^{n-1}} u^{2}=\int_{\mathbb{R}_{+}^{n}}|\nabla i u|^{2} \text { and } \\
& \int_{\mathbb{S}^{n-1}}|u|^{2 \frac{(n-1)}{n-2}}=\int_{\partial \mathbb{R}_{+}^{n}}|i u|^{2 \frac{(n-1)}{n-2}} .
\end{aligned}
$$

In the sequel, we identify the function $H$ and its composition with the stereographic projection $\pi_{q}$. We also identify a point $x$ of $\mathbb{B}^{n}$ and its image by $\pi_{q}$. These facts will be assumed as understood in the sequel.

For $a \in \partial \mathbb{R}_{+}^{n}$ and $\lambda>0$, we define the function

$$
\delta_{(a, \lambda)}(x)=\bar{c} \frac{\lambda^{\frac{n-2}{2}}}{\left(\left(1+\lambda x_{n}\right)^{2}+\lambda^{2}\left|x^{\prime}-a^{\prime}\right|^{2}\right)^{\frac{n-2}{2}}},
$$

where $x \in \mathbb{R}_{+}^{n}$, and $\bar{c}$ is chosen such that $\delta_{a, \lambda}$ satisfies the following equation:

$$
\left\{\begin{array}{l}
\Delta u=0 \quad \text { and } \quad u>0 \quad \text { in } \mathbb{R}_{+}^{n} \\
-\frac{\partial u}{\partial x_{n}}=u^{\frac{n}{n-2}} \text { on } \partial \mathbb{R}_{+}^{n}
\end{array}\right.
$$

Set

$$
\tilde{\delta}_{a, \lambda}=i^{-1}\left(\delta_{(a, \lambda)}\right) .
$$

For $\varepsilon>0, p \in \mathbb{N}^{*}$, let us define

$$
V(p, \varepsilon)=\left\{\begin{array}{l}
u \in \Sigma \quad \text { s.t. } \exists a_{1}, \ldots, a_{p} \in \mathbb{S}^{n-1}, \exists \alpha_{1}, \ldots, \alpha_{p}>0, \\
\exists \lambda_{1}, \ldots, \lambda_{p}>\varepsilon^{-1} \text { with }\left\|u-\sum_{i=1}^{p} \alpha_{i} \tilde{\delta}_{\left(a_{i}, \lambda_{i}\right)}\right\|<\varepsilon, \quad \varepsilon_{i j}<\varepsilon \forall i \neq j, \\
\text { and }\left|\frac{\alpha_{i}^{n-2} H\left(a_{i}\right)}{\alpha_{j}^{n-2} H\left(a_{j}\right)}-1\right|<\varepsilon \quad \forall i, j=1, \ldots, p,
\end{array}\right.
$$

where

$$
\varepsilon_{i j}=\left(\frac{\lambda_{i}}{\lambda_{j}}+\frac{\lambda_{j}}{\lambda_{i}}+\lambda_{i} \lambda_{j}\left|a_{i}-a_{j}\right|^{2}\right)^{\frac{2-n}{2}} .
$$

For $w$, a solution of (1.1), we also define $V(p, \varepsilon, w)$ as

$$
V(p, \varepsilon, w)=\left\{u \in \Sigma / \exists \alpha_{0}>0 \text { s.t. } u-\alpha_{0} w \in V(p, \varepsilon) \text { and }\left|\alpha_{0} J(u)^{\frac{n-1}{2}}-1\right|<\varepsilon\right\} .
$$

If $u$ is a function in $V(p, \varepsilon, w)$, one can find an optimal representation following the ideas introduced in Proposition 5.2 of [25] (see also pp.348-350 of [26]). Namely we have the following proposition. 
Proposition 2.1 For any $p \in \mathbb{N}^{*}$, there is $\varepsilon_{p}>0$ such that if $\varepsilon \leq \varepsilon_{p}$ and $u \in V(p, \varepsilon, w)$, then the minimization problem

$$
\min _{\substack{\alpha_{i}>0, \lambda_{i}>0, a_{i} \in S^{n-1}, h \in T_{w}\left(W_{u}(w)\right)}}\left\|u-\sum_{i=1}^{p} \alpha_{i} \tilde{\delta}_{\left(a_{i}, \lambda_{i}\right)}-\alpha_{0}(w+h)\right\|
$$

has a unique solution $(\alpha, \lambda, a, h)$, up to a permutation.

In particular, we can write $u$ as follows:

$$
u=\sum_{i=1}^{p} \alpha_{i} \tilde{\delta}_{\left(a_{i}, \lambda_{i}\right)}+\alpha_{0}(w+h)+v
$$

where $v$ belongs to $H^{1}\left(\mathbb{B}^{n}\right) \cap T_{w}\left(W_{s}(w)\right)$ and it satisfies $\left(V_{0}\right), T_{w}\left(W_{u}(w)\right)$ and $T_{w}\left(W_{s}(w)\right)$ are the tangent spaces at $w$ of the unstable and stable manifolds of $w$ for a decreasing pseudogradient of $J$ and $\left(V_{0}\right)$ is the following:

$$
\left(V_{0}\right):\left\{\begin{array}{l}
\langle v, \psi\rangle=0 \quad \text { for } \psi \in\left\{\tilde{\delta}_{i}, \frac{\partial \tilde{\delta}_{i}}{\partial \lambda_{i}}, \frac{\partial \tilde{\delta}_{i}}{\partial a_{i}}, i=1, \ldots, p\right\}, \\
\langle v, w\rangle=0, \\
\langle v, h\rangle=0 \quad \text { for all } h \in T_{w} W_{u}(w) .
\end{array}\right.
$$

Here, $\tilde{\delta}_{i}=\tilde{\delta}_{\left(a_{i}, \lambda_{i}\right)}$ and $\langle\cdot, \cdot\rangle$ denotes the scalar product defined on $H^{1}\left(\mathbb{B}^{n}\right)$ by

$$
\langle u, v\rangle=\int_{\mathbb{B}^{n}} \nabla u \nabla v d v_{g_{0}}+\frac{n-2}{2} \int_{\mathbb{S}^{n-1}} u v d \sigma_{g_{0}} .
$$

Notice that Proposition 2.1 is also true if we take $w=0$, and therefore, $h=0$ and $u$ in $V(p, \varepsilon)$.

The failure of the Palais-Smale condition can be characterized taking into account the uniqueness result of $\mathrm{Li}$ and $\mathrm{Zhu}$ [27]. Following the ideas introduced in [20], we have the following proposition.

Proposition 2.2 Let $\left(u_{k}\right)$ be a sequence in $\Sigma^{+}$such that $J\left(u_{k}\right)$ is bounded and $\partial J\left(u_{k}\right)$ goes to zero. Then there exist an integer $p \in \mathbb{N}^{*}$, a sequence $\left(\varepsilon_{K}\right)>0, \varepsilon_{k}$ tends to zero, and an extracted subsequence of $u_{k}$ 's, again denoted by $\left(u_{k}\right)$, such that $u_{k} \in V\left(p, \varepsilon_{k}, w\right)$, where $w$ is zero or a solution of (1.1).

Now, arguing as in [26] (pp.326, 327 and 334), we have the following Morse lemma which completely gets rid of the $v$-contributions and shows that it can be neglected with respect to the concentration phenomenon.

Proposition 2.3 There is a $\mathcal{C}^{1}$-map which to each $\left(\alpha_{i}, a_{i}, \lambda_{i}, h\right)$ such that $\sum_{i=1}^{p} \alpha_{i} \tilde{\delta}_{\left(a_{i}, \lambda_{i}\right)}+$ $\alpha_{0}(w+h)$ belongs to $V(p, \varepsilon, w)$ associates $\bar{v}=\bar{v}(\alpha, a, \lambda, h)$ such that $\bar{v}$ is unique and satisfies

$$
J\left(\sum_{i=1}^{p} \alpha_{i} \tilde{\delta}_{\left(a_{i}, \lambda_{i}\right)}+\alpha_{0}(w+h)+\bar{v}\right)=\min _{v \in\left(V_{0}\right)}\left\{J\left(\sum_{i=1}^{p} \alpha_{i} \tilde{\delta}_{\left(a_{i}, \lambda_{i}\right)}+\alpha_{0}(w+h)+v\right)\right\} .
$$


Moreover, there exists a change of variables $v-\bar{v} \rightarrow V$ such that

$$
J\left(\sum_{i=1}^{p} \alpha_{i} \tilde{\delta}_{\left(a_{i}, \lambda_{i}\right)}+\alpha_{0}(w+h)+v\right)=J\left(\sum_{i=1}^{p} \alpha_{i} \tilde{\delta}_{\left(a_{i}, \lambda_{i}\right)}+\alpha_{0}(w+h)+\bar{v}\right)+\|V\|^{2} .
$$

We notice that in the $V$ variable we define a pseudo-gradient by setting

$$
\frac{\partial V}{\partial s}=-\mu V
$$

where $\mu$ is a very large constant. Then at $s=1, V(s)=e^{-\mu s} V(0)$ is very small, as we wish. This shows that in order to define our deformation, we can work as if $V$ was zero. The deformation extends immediately with the same properties to a neighborhood of zero in the $V$ variable.

Definition 2.4 A critical point at infinity of $J$ on $\Sigma^{+}$is a limit of a flow line $u(s)$ of the equation

$$
\left\{\begin{array}{l}
\frac{\partial u}{\partial s}=-\partial J(u(s)) \\
u(0)=u_{0}
\end{array}\right.
$$

such that $u(s)$ remains in $V(p, \varepsilon(s), w)$ for $s \geq s_{0}$. Here $w$ is either zero or a solution of (1.1) and $\varepsilon(s)$ is some positive function tending to zero when $s \rightarrow+\infty$. Using Proposition 2.1, $u(s)$ can be written as

$$
u(s)=\sum_{i=1}^{p} \alpha_{i}(s) \delta_{\left(a_{i}(s), \lambda_{i}(s)\right)}+\alpha_{0}(s)(w+h(s))+v(s) .
$$

Denoting $\tilde{\alpha}_{i}:=\lim _{s \rightarrow+\infty} \alpha_{i}(s), \tilde{y}_{i}:=\lim _{s \rightarrow+\infty} a_{i}(s)$, we denote by

$$
\sum_{i=1}^{p} \tilde{\alpha}_{i} \delta_{\left(\tilde{y}_{i}, \infty\right)}+\tilde{\alpha}_{0} w \quad \text { or } \quad\left(\tilde{y}_{1}, \ldots, \tilde{y}_{p}, w\right)_{\infty}
$$

such a critical point at infinity. If $w \neq 0$, it is called of $w$-type or mixed type.

With such a critical point at infinity, stable and unstable manifolds are associated. These manifolds can be easily described once a Morse-type reduction is performed; see [26] (pp.356-357).

\subsection{Expansion of the gradient of the functional}

In this subsection we recall some useful expansions of the gradient of the functional $J$. These expansions are extracted from ([22], Appendix A).

Proposition 2.5 For any $u=\sum_{i=1}^{p} \alpha_{i} \tilde{\delta}_{i} \in V(p, \varepsilon)$, we have the following expansions:

$$
\begin{aligned}
\left\langle\nabla J(u), \lambda_{i} \frac{\partial \tilde{\delta}_{i}}{\partial \lambda_{i}}\right\rangle= & 2 J(u)\left[-c_{1} \sum_{j \neq i} \alpha_{j} \lambda_{i} \frac{\partial \varepsilon_{i j}}{\partial \lambda_{i}}+J(u)^{\frac{n-1}{n-2}} \frac{n-2}{n-1} \alpha_{i}^{\frac{n}{n-2}} \frac{c_{2} \Delta H\left(a_{i}\right)}{\lambda_{i}^{2}}\right] \\
& +o\left(\frac{1}{\lambda_{i}^{2}}+\sum_{j \neq i} \varepsilon_{i j}\right),
\end{aligned}
$$




$$
\begin{aligned}
\left\langle\nabla J(u), \frac{1}{\lambda_{i}} \frac{\partial \tilde{\delta}_{i}}{\partial a_{i}}\right\rangle= & 2 J(u)\left[-c_{1} \sum_{j \neq i} \frac{\alpha_{j}}{\lambda_{i}} \frac{\partial \varepsilon_{i j}}{\partial a_{i}}-J(u)^{\frac{n-1}{n-2}} c_{3} \alpha_{i}^{\frac{n}{n-2}} \frac{\nabla H\left(a_{i}\right)}{\lambda_{i}}\right] \\
& +o\left(\sum_{i \neq j} \varepsilon_{i j}\right)+O\left(\frac{1}{\lambda_{i}^{2}}\right)
\end{aligned}
$$

where $c_{1}, c_{2}$ and $c_{3}$ are three positive constants.

Proposition 2.6 For each $u=\sum_{i=1}^{p} \alpha_{i} \tilde{\delta}_{i} \in V(p, \varepsilon)$, if $a_{i}$ is close to a critical point $y$ of $H$ satisfying $(\mathfrak{f})_{\beta}$, then we have the following expansions:

$$
\begin{aligned}
\left\langle\nabla J(u), \frac{1}{\lambda_{i}} \frac{\partial \tilde{\delta}_{i}}{\partial\left(a_{i}\right)_{k}}\right\rangle= & -2 J(u)^{\frac{2 n-3}{n-2}} \alpha_{i}^{\frac{n}{n-2}} \frac{1}{\lambda_{i}^{\beta}} \int_{\mathbb{R}^{n-1}} \frac{b_{k}\left|x_{k}+\lambda_{i}\left(a_{i}\right)_{k}\right|^{\beta} x_{k}}{\left(1+|x|^{2}\right)^{n}} d x \\
& +O\left(\sum_{i \neq j} \varepsilon_{i j}\right)+o\left(\frac{1}{\lambda_{i}^{\beta}}\right), \\
\left\langle\nabla J(u), \lambda_{i} \frac{\partial \tilde{\delta}_{i}}{\partial \lambda_{i}}\right\rangle= & c \frac{\sum b_{j}}{\lambda_{i}^{\beta}} \int_{\mathbb{R}^{n-1}}\left|x_{j}+\lambda_{i}\left(a_{i}\right)_{j}\right|^{\beta} \frac{1-|x|^{2}}{\left(1+|x|^{2}\right)^{n}} d x \\
& -2 c_{1} J(u) \sum_{j \neq i} \alpha_{j} \lambda_{i} \frac{\partial \varepsilon_{i j}}{\partial \lambda_{i}}+o\left(\frac{1}{\lambda_{i}^{\beta}}+\sum_{j \neq i} \varepsilon_{i j}\right),
\end{aligned}
$$

where $\left(a_{i}\right)_{k}$ is the kth component of $a_{i}$ in some geodesic normal coordinates system. Furthermore, if we assume that $\lambda_{i}\left|a_{i}\right| \leq \rho$ is a small positive constant, then

$$
\left\langle\nabla J(u), \lambda_{i} \frac{\partial \tilde{\delta}_{i}}{\partial \lambda_{i}}\right\rangle=-2 c_{1} J(u) \sum_{j \neq i} \alpha_{j} \lambda_{i} \frac{\partial \varepsilon_{i j}}{\partial \lambda_{i}}+c \frac{\sum b_{j}}{\lambda_{i}^{\beta}}+o\left(\frac{1}{\lambda_{i}^{\beta}}+\sum_{j \neq i} \varepsilon_{i j}\right) .
$$

\section{Characterization of critical points at infinity}

This section is devoted to the characterization of critical points at infinity in $V(p, \varepsilon), p \geq$ 1 , under $\beta$-flatness condition with $n-2 \leq \beta<n-1$. This characterization is obtained through the construction of a suitable pseudo-gradient at infinity, for which the PalaisSmale condition is satisfied along the decreasing flow lines as long as these flow lines do not enter the neighborhood of a finite number of critical points $y_{i}, i=1, \ldots, p$, of $H$ such that $\left(y_{1}, \ldots, y_{p}\right) \in C_{n-2}^{+} \cup\left(\mathcal{K}^{+} \backslash \mathcal{K}_{n-2}\right)$. Recall that the construction was done in $V(1, \varepsilon)$.

Theorem 3.1 ([22], Proposition 5.2) There exists a pseudo-gradient $\widetilde{W}_{2}$ in $V(1, \varepsilon)$ so that the following holds. There is a positive constant $c>0$ independent $u=\alpha \tilde{\delta}_{a \lambda} \in V(1, \varepsilon)$ such that

(i) $\left\langle\partial J(u), \widetilde{W}_{2}(u)\right\rangle \leq c\left(\frac{1}{\lambda^{\beta}}+\frac{|\nabla H(a)|}{\lambda}\right)$,

(ii) $\left\langle\partial J(u+\bar{v}), \widetilde{W}_{2}(u)+\frac{\partial \bar{v}}{\partial\left(\alpha_{i}, a_{i}, \lambda_{i}\right)}\left(\widetilde{W}_{2}(u)\right)\right\rangle \leq c\left(\frac{1}{\lambda^{\beta}}+\frac{|\nabla H(a)|}{\lambda}\right)$.

Furthermore, $\left|\widetilde{W}_{2}\right|$ is bounded and the only case where $\lambda$ is not bounded is where $a \in B(y, \rho)$, $y \in \mathcal{K}^{+}$.

Next, we give the characterization of the critical points at infinity in $V(p, \varepsilon), p \geq 2$. We have the following main result. 
Theorem 3.2 Let $\beta:=\max \{\beta(y) / y \in \mathcal{K}\}$. For $p \geq 2$, there exists a pseudo-gradient $\widetilde{W}_{1}$ in $V(p, \varepsilon)$ so that the following holds.

There exists a constant $c>0$ independent of $u=\sum_{i=1}^{p} \alpha_{i} \tilde{\delta}_{i} \in V(p, \varepsilon)$ so that

(i) $\left\langle\partial J(u), \widetilde{W}_{1}(u)\right\rangle \leq-c\left(\sum_{i=1}^{p} \frac{1}{\lambda_{i}^{\beta}}+\sum_{i=1}^{p} \frac{\nabla H\left(a_{i}\right)}{\lambda_{i}}+\sum_{j \neq i} \varepsilon_{i j}\right)$,

(ii) $\left\langle\partial J(u+\bar{v}), \widetilde{W}_{1}(u)+\frac{\partial \bar{v}}{\partial\left(\alpha_{i}, a_{i}, \lambda_{i}\right)}\left(\widetilde{W}_{1}(u)\right)\right\rangle \leq-c\left(\sum_{i=1}^{p} \frac{1}{\lambda_{i}^{\beta}}+\sum_{i=1}^{p} \frac{\nabla H\left(a_{i}\right)}{\lambda_{i}}+\sum_{j \neq i} \varepsilon_{i j}\right)$.

Furthermore, $\left|\widetilde{W}_{1}\right|$ is bounded and the only case where the maximum of the $\lambda_{i}$ 's is not bounded is when $a_{i} \in B\left(y_{l_{i}}, \rho\right) \forall i=1, \ldots, p$ with $\left(y_{l_{1}}, \ldots, y_{l_{p}}\right) \in \mathcal{C}_{n-2}^{+}$.

We will prove Theorem 3.2 later. Now we state two results which deal with two specific cases of Theorem 3.2. Let

$$
\begin{aligned}
& V_{1}(p, \varepsilon)=\left\{u=\sum_{i=1}^{p} \alpha_{i} \tilde{\delta}_{i} \in V(p, \varepsilon) \text { s.t. } a_{i} \in B\left(y_{l_{i}}, \rho\right), y_{l_{i}} \in \mathcal{K} \backslash \mathcal{K}_{n-2} \forall i=1, \ldots, p\right\}, \\
& V_{2}(p, \varepsilon)=\left\{u=\sum_{i=1}^{p} \alpha_{i} \tilde{\delta}_{i} \in V(p, \varepsilon) \text { s.t. } a_{i} \in B\left(y_{l_{i}}, \rho\right), y_{l_{i}} \in \mathcal{K}_{n-2} \forall i=1, \ldots, p\right\} .
\end{aligned}
$$

We then have the following.

Proposition 3.3 ([22], Proposition 5.1) For $p \geq 2$, there exists a pseudo-gradient $W_{1}$ in $V_{1}(p, \varepsilon)$ so that the following holds.

There exists $c>0$ independent of $u=\sum_{i=1}^{p} \alpha_{i} \tilde{\delta}_{i} \in V_{1}(p, \varepsilon)$ such that

$$
\left\langle\partial J(u), W_{1}(u)\right\rangle \leq-c\left(\sum_{i=1}^{p} \frac{1}{\lambda_{i}^{\beta}}+\sum_{i \neq j} \varepsilon_{i j}+\sum_{i=1}^{p} \frac{\left|\nabla H\left(a_{i}\right)\right|}{\lambda_{i}}\right) .
$$

Furthermore, $\left|W_{1}\right|$ is bounded and the maximum of $\lambda_{i}$ 's decreases along the flow lines of $W_{1}$.

Proposition 3.4 For $p \geq 2$, there exists a pseudo-gradient $W_{2}$ in $V_{2}(p, \varepsilon)$ such that $\forall u=$ $\sum_{i=1}^{p} \alpha_{i} \tilde{\delta}_{i} \in V_{2}(p, \varepsilon)$, we have

$$
\left\langle\partial J(u), W_{2}(u)\right\rangle \leq-c\left(\sum_{i=1}^{p} \frac{1}{\lambda_{i}^{n-2}}+\sum_{i \neq j} \varepsilon_{i j}+\sum_{i=1}^{p} \frac{\left|\nabla H\left(a_{i}\right)\right|}{\lambda_{i}}\right),
$$

where $c$ is a positive constant independent of $u$. Furthermore, we have $\left|W_{2}\right|$ is bounded and the only case where the maximum of $\lambda_{i}$ 's is not bounded is when $a_{i} \in B\left(y_{l_{i}}, \rho\right) \forall i=1, \ldots, p$ with $\left(y_{l_{1}}, \ldots, y_{l_{p}}\right) \in \mathcal{C}_{n-2}^{+}$.

Before giving the proof of Theorem 3.2 and Proposition 3.4, we state the following notation extracted from [22], Section 4.

Let $u=\sum_{i=1}^{p} \alpha_{i} \tilde{\delta}_{\left(a_{i}, \lambda_{i}\right)} \in V(p, \varepsilon)$. For simplicity, if $a_{i}$ is close to a critical point $y_{l_{i}}$, we assume that the critical point is zero, so we confuse $a_{i}$ with $\left(a_{i}-y_{l_{i}}\right)$. Now, let $i \in\{1, \ldots, p\}$ 
and let $M_{1}$ be a positive large constant. We say that

$$
i \in L_{1} \quad \text { if } \lambda_{i}\left|a_{i}\right| \leq M_{1}
$$

and we say that

$$
i \in L_{2} \quad \text { if } \lambda_{i}\left|a_{i}\right|>M_{1} \text {. }
$$

For each $i \in\{1, \ldots, p\}$, we define the following vector fields:

$$
Z_{i}(u)=\alpha_{i} \lambda_{i} \frac{\partial \tilde{\delta}_{i}}{\partial \lambda_{i}}
$$

and

$$
X_{i}=\alpha_{i} \sum_{k=1}^{n-1} \frac{1}{\lambda_{i}} \frac{\partial \tilde{\delta}_{\left(a_{i}, \lambda_{i}\right)}}{\partial\left(a_{i}\right)_{k}} \int_{\mathbb{R}^{n-1}} b_{k} \frac{\left|x_{k}+\lambda_{i}\left(a_{i}\right)_{k}\right|^{\beta}}{\left(1+\lambda_{i}\left|\left(a_{i}\right)_{k}\right|\right)^{\beta-1}} \frac{x_{k}}{\left(1+|x|^{2}\right)^{n}} d x,
$$

where $\left(a_{i}\right)_{k}$ is the $k$ th component of $a_{i}$ in some geodesic normal coordinates system.

We claim that $X_{i}$ is bounded. Indeed, the claim is trivial if $i \in L_{1}$. If $i \in L_{2}$, by elementary computation, we have the following estimate:

$$
\begin{aligned}
\int_{\mathbb{R}^{n-1}} \frac{\left|x_{k}+\lambda_{i}\left(a_{i}\right)_{k}\right|^{\beta} x_{k}}{\left(1+|x|^{2}\right)^{n}} d x & =\left(\lambda_{i}\left|\left(a_{i}\right)_{k}\right|\right)^{\beta} \int_{\mathbb{R}^{n-1}}\left|1+\frac{x_{k}}{\lambda_{i}\left(\left(a_{i}\right)_{k}\right)}\right|^{\beta} \frac{x_{k}}{\left(1+|x|^{2}\right)^{n}} d x \\
& =c\left(\text { signe } \lambda_{i}\left(a_{i}\right)_{k}\right)\left(\lambda_{i}\left|\left(a_{i}\right)_{k}\right|\right)^{\beta-1}(1+o(1))
\end{aligned}
$$

for any $k, 1 \leq k \leq n-1$ such that $\lambda_{i}\left|\left(a_{i}\right)_{k}\right|>\frac{M_{1}}{\sqrt{n-1}}$. Hence, our claim is valid.

Let $k_{i}$ be an index such that

$$
\left|\left(a_{i}\right)_{k_{i}}\right|=\max _{1 \leq j \leq n}\left|\left(a_{i}\right)_{j}\right|
$$

It easy to see that if $i \in L_{2}$ then $\lambda_{i}\left|\left(a_{i}\right)_{k_{i}}\right|>\frac{M_{1}}{\sqrt{n-1}}$.

Proof of Theorem 3.2 Thanks to Propositions 3.3 and 3.4 and in order to complete the construction of the pseudo-gradient $\widetilde{W}_{1}$ suggested in Theorem 3.2 , it only remains to focus attention on the two following sets of $V(p, \varepsilon)$.

Subset 1: We consider here the case of $u=\sum_{i=1}^{p} \alpha_{i} \tilde{\delta}_{i}=\sum_{i \in I_{1}} \alpha_{i} \tilde{\delta}_{i}+\sum_{i \in I_{2}} \alpha_{i} \tilde{\delta}_{i}$ such that

$$
I_{1} \neq \varnothing, \quad I_{2} \neq \varnothing, \quad \sum_{i \in I_{1}} \alpha_{i} \tilde{\delta}_{i} \in V_{1}\left(\sharp I_{1}, \varepsilon\right) \quad \text { and } \quad \sum_{i \in I_{2}} \alpha_{i} \tilde{\delta}_{i} \in V_{2}\left(\sharp I_{2}, \varepsilon\right) .
$$

Without loss of generality, we can assume that $\lambda_{1} \leq \cdots \leq \lambda_{p}$. Let

$$
I=\{1\} \cup\left\{i, 1 \leq i \leq p \text {, s.t. } \lambda_{i} \leq \tilde{M} \lambda_{1}\right\},
$$

where $\widetilde{M}$ is a positive constant large enough. Now, let $I^{\prime}=I_{1} \cap I$, we distinguish three cases. 
Case 1. $I^{\prime}=\varnothing$. Then $I \subset I_{2}$, so we derive

$$
u=u_{1}+u_{2} \quad \text { where } u_{1}=\sum_{i \in I} \alpha_{i} \tilde{\delta}_{i} \text { and } u_{2}=\sum_{i \notin I} \alpha_{i} \tilde{\delta}_{i} .
$$

Observe that $u_{1} \in V_{2}(\sharp I, \varepsilon)$, we can apply then the vector field $W_{2}$ defined in Proposition 3.4 in this set. We obtain

$$
\left\langle\partial J(u), W_{2}(u)\right\rangle \leq-c\left(\sum_{i \in I} \frac{1}{\lambda_{i}^{n-2}}+\sum_{i \neq j ; i, j \in I} \varepsilon_{i j}+\sum_{i \in I} \frac{\left|\nabla H\left(a_{i}\right)\right|}{\lambda_{i}}\right)+O\left(\sum_{i \in I, j \notin I} \varepsilon_{i j}\right) .
$$

For the indices $i$ such that $i \notin I$, we apply the vector field $\sum_{i \notin I}-2^{i} Z_{i}(u)$.

Observe that in $V_{1}(p, \varepsilon) \cup V_{2}(p, \varepsilon)$ and under assumption $(\mathfrak{f})_{\beta}$, we have

$$
\Delta H(x) \cong \sum_{k=1}^{n-1} b_{k} \beta(\beta-1)\left|x_{k}\right|^{\beta-2}
$$

Then

$$
\left|\Delta H\left(a_{i}\right)\right| \leq c \sum_{k=1}^{n-1}\left|\left(a_{i}\right)_{k}\right|^{\beta-2} \leq c(n-1)\left|\left(a_{i}\right)_{k_{i}}\right|^{\beta-2},
$$

where $k_{i}$ is defined in (3.4). Hence, we derive that

$$
\frac{\left|\Delta H\left(a_{i}\right)\right|}{\lambda_{i}^{2}}=O\left(\frac{\left|\left(a_{i}\right)_{k_{i}}\right|^{\beta-2}}{\lambda_{i}^{2}}\right) .
$$

Using Proposition 2.6 and (3.6), we obtain

$$
\begin{aligned}
\left\langle\partial J(u), \sum_{i \notin I}-2^{i} Z_{i}(u)\right\rangle \leq & c \sum_{j \neq i, i \notin I} 2^{i} \lambda_{i} \frac{\partial \varepsilon_{i j}}{\partial \lambda_{i}}+O\left(\sum_{i \notin I, i \in L_{2}} \frac{\left|\left(a_{i}\right)_{k_{i}}\right|^{\beta-2}}{\lambda_{i}^{2}}\right) \\
& +O\left(\sum_{i \notin I, i \in L_{1}} \frac{1}{\lambda_{i}^{\beta}}\right),
\end{aligned}
$$

where $k_{i}$ is defined in (3.4). An easy calculation yields

$$
\lambda_{i} \frac{\partial \varepsilon_{i j}}{\partial \lambda_{i}} \leq-c \varepsilon_{i j} \quad \text { if } \lambda_{i} \geq \lambda_{j} \text { or } \lambda_{i} \sim \lambda_{j} \text { or }\left|a_{i}-a_{j}\right| \geq \delta_{0}>0 .
$$

In addition, it is easy to see that for $i \in L_{2}$, we have

$$
\frac{\left|\left(a_{i}\right)_{k_{i}}\right|^{\beta-2}}{\lambda_{i}^{2}}=o\left(\frac{\left|\left(a_{i}\right)_{k_{i}}\right|^{\beta-1}}{\lambda_{i}}\right) \text { taking } M_{1} \text { large enough. }
$$

Observe now that for $i<j$, we have

$$
2^{i} \lambda_{i} \frac{\partial \varepsilon_{i j}}{\partial \lambda_{i}}+2^{j} \lambda_{j} \frac{\partial \varepsilon_{i j}}{\partial \lambda_{j}} \leq-c \varepsilon_{i j}
$$


These estimates with (3.10) yield

$$
\begin{aligned}
\left\langle\partial J(u), \sum_{i \notin I}-2^{i} Z_{i}(u)\right\rangle \leq & -c \sum_{i \neq j, i \notin I} \varepsilon_{i j}+O\left(\sum_{i \notin I, i \in L_{1}} \frac{1}{\lambda_{i}^{\beta}}\right) \\
& +o\left(\sum_{i \notin I, i \in L_{2}} \frac{\left|\left(a_{i}\right)_{k_{i}}\right|^{\beta-1}}{\lambda_{i}}\right) .
\end{aligned}
$$

From another part, by Proposition 2.6, we find that

$$
\begin{aligned}
& \left\langle\partial J(u), \sum_{i \notin I, i \in L_{2}} X_{i}(u)\right\rangle \\
& \leq-c \sum_{i \notin I, i \in L_{2}} \frac{1}{\lambda_{i}^{\beta}}\left(\int_{\mathbb{R}^{n-1}} b_{k_{i}} \frac{\left|x_{k_{i}}+\lambda_{i}\left(a_{i}\right)_{k_{i}}\right|^{\beta}}{\left(1+\lambda_{i}\left|\left(a_{i}\right)_{k_{i}}\right|\right)^{\frac{\beta-1}{2}}} \frac{x_{k_{i}}}{\left(1+|x|^{2}\right)^{n}} d x\right)^{2} \\
& \quad+O\left(\sum_{i \neq j, i \in I, i \in L_{2}} \varepsilon_{i j}\right) .
\end{aligned}
$$

Identity (3.3) implies

$$
\left\langle\partial J(u), \sum_{i \notin I, i \in L_{2}} X_{i}(u)\right\rangle \leq-c \sum_{i \notin I, i \in L_{2}} \frac{\left|\left(a_{i}\right)_{k_{i}}\right|^{\beta-1}}{\lambda_{i}}+O\left(\sum_{i \neq j, i \in I, i \in L_{2}} \varepsilon_{i j}\right) .
$$

Observe that since $a_{i} \in B\left(y_{j_{i}}, \rho\right)$ and $H$ satisfies $(\mathfrak{f})_{\beta}$, we have $\left|\nabla H\left(a_{i}\right)\right| \sim\left|\left(a_{i}\right)_{k_{i}}\right|^{\beta-1}$. Thus,

$$
\begin{aligned}
\left\langle\partial J(u), \sum_{i \notin I, i \in L_{2}} X_{i}(u)\right\rangle \leq & -c\left(\sum_{i \notin I, i \in L_{2}} \frac{\left|\nabla H\left(a_{i}\right)\right|}{\lambda_{i}}+\sum_{i \notin I, i \in L_{2}} \frac{\left|\left(a_{i}\right)_{k_{i}}\right|^{\beta-1}}{\lambda_{i}}\right) \\
& +O\left(\sum_{i \neq j, i \notin I, i \in L_{2}} \varepsilon_{i j}\right) .
\end{aligned}
$$

Let $W_{3}^{1}=\sum_{i \notin I}-2^{i} Z_{i}+m_{1} \sum_{i \notin I, i \in L_{2}} X_{i}$, where $m_{1}$ is a positive constant small enough. From (3.11) and (3.13), we find that

$$
\begin{aligned}
\left\langle\partial J(u), W_{3}^{1}(u)\right\rangle \leq & -c\left(\sum_{i \neq j, i \notin I} \varepsilon_{i j}+\sum_{i \notin I, i \in L_{2}} \frac{\left|\nabla H\left(a_{i}\right)\right|}{\lambda_{i}}\right) \\
& +O\left(\sum_{i \notin I, i \in L_{1}} \frac{1}{\lambda_{i}^{\beta}}\right) .
\end{aligned}
$$

Observe that $\forall i \notin I$

$$
\frac{1}{\lambda_{i}^{\beta}}=o\left(\frac{1}{\lambda_{1}^{n-2}}\right) \text { for } \tilde{M} \text { large enough. }
$$

Let, in this case, $W_{3}$ be the following vector field.

$$
W_{3}:=W_{3}^{1}+m_{1} W_{2}
$$


From (3.5) and (3.14), we obtain

$$
\left\langle\partial J(u), W_{3}(u)\right\rangle \leq-c\left(\sum_{i=1}^{p} \frac{1}{\lambda_{i}^{\beta}}+\sum_{i \neq j} \varepsilon_{i j}+\sum_{i=1}^{p} \frac{\left|\nabla H\left(a_{i}\right)\right|}{\lambda_{i}}\right)
$$

since for $i \notin I$ and $i \in L_{1}$, we have $\frac{\left|\nabla H\left(a_{i}\right)\right|}{\lambda_{i}}=O\left(\frac{1}{\lambda_{i}^{\beta}}\right)=o\left(\frac{1}{\lambda_{1}^{n-2}}\right)$.

Case 2. $I=I^{\prime}=\{1\}, a_{1}$ is then close to $y_{l_{1}} \in \mathcal{K}^{i} \backslash \mathcal{K}_{n-2}$, we define

$$
\widetilde{Z}_{1}=-\left(\sum_{k=1}^{n} b_{k}\right) \psi\left(\lambda_{1}\left|a_{1}\right|\right) Z_{1}
$$

where $\psi$ is a cutoff function defined by $\psi(t)=1$ if $|t| \leq \delta$ and $\psi(t)=0$ if $|t| \geq 2 \delta$ ( $\delta$ is a positive constant small enough). Using Proposition 2.6, we have

$$
\begin{aligned}
\left\langle\partial J(u), \widetilde{Z}_{1}(u)+X_{1}(u)\right\rangle \leq & -\frac{c}{\lambda_{1}^{\beta}}\left(\int_{\mathbb{R}^{n-1}} b_{k} \frac{\left|x_{k_{i}}+\lambda_{i}\left(a_{1}\right)_{k_{i}}\right|^{\beta}}{\left(1+\lambda_{i}\left|\left(a_{1}\right)_{k_{i}}\right|\right)^{\frac{\beta-1}{2}}} \frac{x_{k_{i}}}{\left(1+|x|^{2}\right)^{n}} d x\right)^{2} \\
& -\psi\left(\lambda_{1}\left|a_{1}\right|\right)\left(\sum_{i=1}^{n} b_{k}\right)^{2} \frac{c_{3}}{\lambda_{1}^{\beta}}+O\left(\sum_{j \neq 1} \varepsilon_{1 j}\right) .
\end{aligned}
$$

We need to prove the following claim:

$$
\left\langle\partial J(u), \widetilde{Z}_{1}(u)+X_{1}(u)\right\rangle \leq-c\left(\frac{1}{\lambda_{1}^{\beta}}+\frac{\left|\nabla H\left(a_{1}\right)\right|}{\lambda_{1}}\right)+O\left(\sum_{j \neq 1} \varepsilon_{1 j}\right) .
$$

Observe that if $i \in L_{2}$ then using (3.3) we can make appear $\frac{1}{\lambda_{1}^{\beta}}$ and $\frac{\left|\nabla H\left(a_{1}\right)\right|}{\lambda_{1}}$ in the upper bound of (3.16) and our claim follows in this case.

Now, if $\lambda_{1}\left|a_{1}\right|<\delta$, then we have $\psi\left(\lambda_{1}\left(a_{1}\right)\right)=1$ and $\frac{\left|\nabla H\left(a_{1}\right)\right|}{\lambda_{1}} \sim \frac{\left|\left(a_{1}\right)_{k_{i}}\right|^{\beta-1}}{\lambda_{1}}$ is small with respect to $\frac{1}{\lambda_{1}^{\beta}}$. Thus (3.17) holds in this case. Finally, if $\lambda_{1}\left|a_{1}\right|$ is bounded below and above, in this case, using elementary calculation, we have

$$
\left(\int_{\mathbb{R}^{n-1}} b_{k_{i}} \frac{\left|x_{k_{i}}+\lambda_{i}\left(a_{1}\right)_{k_{i}}\right|^{\beta}}{\left(1+\lambda_{i}\left|\left(a_{1}\right)_{k_{i}}\right|\right)^{\frac{\beta-1}{2}}} \frac{x_{k_{i}}}{\left(1+|x|^{2}\right)^{n}} d x\right)^{2} \geq c>0 .
$$

We then obtain (3.17) and hence our claim is valid. This with (3.6), (3.10) and Proposition 2.6 yields

$$
\begin{aligned}
& \left\langle\partial J(u), \sum_{i \geq 2}-2^{i} Z_{i}(u)+m_{1}\left(\widetilde{Z}_{1}(u)+X_{1}(u)\right)\right\rangle \\
& \leq-c\left(\sum_{i \neq j} \varepsilon_{i j}+\frac{1}{\lambda_{1}^{\beta}}+\frac{\left|\nabla H\left(a_{1}\right)\right|}{\lambda_{1}}\right)+O\left(\sum_{i \geq 2, i \in L_{2}} \frac{\left|\left(a_{i}\right)_{k_{i}}\right|^{\beta-2}}{\lambda_{i}^{2}}\right) \\
& \quad+O\left(\sum_{i \geq 2, i \in L_{1}} \frac{1}{\lambda_{i}^{\beta}}\right)
\end{aligned}
$$


for $m_{1}$ a small positive constant. Observe that $\forall i \geq 2$, we have $i \notin I$, so it is easy to see that $\frac{1}{\lambda_{i}^{n-2}}=o\left(\varepsilon_{1 i}\right)$. Taking $\tilde{M}$ large enough, we derive that

$$
\frac{1}{\lambda_{i}^{\beta}}=o\left(\varepsilon_{1 i}\right) \quad \forall i \geq 2
$$

Let, in this case,

$$
W_{3}=\sum_{i \geq 2}-2^{i} Z_{i}+m_{1}\left(\widetilde{Z}_{1}+X_{1}+\sum_{i \geq 2, i \in L_{2}} X_{i}\right) .
$$

Using Proposition 2.6 and estimates (3.3), (3.9), (3.19) and (3.20), we find that

$$
\left\langle\partial J(u), W_{3}(u)\right\rangle \leq-c\left(\sum_{i=1}^{p} \frac{1}{\lambda_{i}^{\beta}}+\sum_{i=1}^{p} \frac{\left|\nabla H\left(a_{i}\right)\right|}{\lambda_{i}}+\sum_{i \neq j} \varepsilon_{i j}\right) .
$$

Case 3. $I^{\prime} \neq \varnothing$ and $\sharp I \geq 2$. Applying the above estimates, we get

$$
\begin{aligned}
\left\langle\partial J(u), \sum_{i \in I^{\prime}}-Z_{i}\right\rangle \leq & -c \sum_{i \neq j, i \in I^{\prime}, j \in I} \varepsilon_{i j}+O\left(\sum_{i \in I^{\prime}, j \notin I} \varepsilon_{i j}\right) \\
& +O\left(\sum_{i \in I^{\prime}, i \in L_{2}} \frac{\left|\left(a_{i}\right)_{k_{i}}\right|^{\beta-2}}{\lambda_{i}^{2}}\right)+O\left(\sum_{i \in I^{\prime}, i \in L_{1}} \frac{1}{\lambda_{i}^{\beta}}\right), \\
\left\langle\partial J(u), \sum_{i \in I \backslash I^{\prime}}-Z_{i}\right\rangle \leq & -c \sum_{i \neq j, i \in I \backslash I^{\prime}, j \in I} \varepsilon_{i j}+O\left(\sum_{i \in I \backslash I^{\prime}, j \notin I} \varepsilon_{i j}\right) \\
& +O\left(\sum_{i \in I \backslash I^{\prime}, i \in L_{1}} \frac{1}{\lambda_{i}^{\beta}}\right)+O\left(\sum_{i \in I \backslash I^{\prime}, i \in L_{2}} \frac{\left|\left(a_{i}\right)_{k_{i}}\right|^{\beta-2}}{\lambda_{i}^{2}}\right), \\
\left\langle\partial J(u), \sum_{i \notin I}-2^{i} Z_{i}\right\rangle \leq & -c \sum_{i \notin I, j \neq i} \varepsilon_{i j}+O\left(\sum_{i \notin I, i \in L_{2}} \frac{\left|\left(a_{i}\right)_{k_{i}}\right|^{\beta-2}}{\lambda_{i}^{2}}\right) \\
& +O\left(\sum_{i \notin I, i \in L_{1}} \frac{1}{\lambda_{i}^{\beta}}\right) .
\end{aligned}
$$

Observe that if $i \notin I$, we have $\frac{1}{\lambda_{i}^{\beta}}=o\left(\varepsilon_{1 i}\right)$, if $i \in I^{\prime}$, we have $\frac{1}{\lambda_{i}^{\beta}}=o\left(\varepsilon_{i j}\right)$, here $j \neq i \in I$, and if $i \in I \backslash I^{\prime}$, we have $\frac{1}{\lambda_{i}^{n-2}}=O\left(\varepsilon_{i j}\right)$, where $j \neq i \in I$. Thus, let in this case

$$
W_{3}=\sum_{i \in I^{\prime}}\left(-Z_{i}\right)-\sum_{i \in I} 2^{i} Z_{i}+m_{1}\left(\sum_{i \in I \backslash I^{\prime}}\left(-Z_{i}\right)+\sum_{i=1, i \in L_{2}}^{p} X_{i}\right) .
$$

We then have

$$
\left\langle\partial J(u), W_{3}(u)\right\rangle \leq-c\left(\sum_{i=1}^{p} \frac{1}{\lambda_{i}^{\beta}}+\sum_{i=1}^{p} \frac{\left|\nabla H\left(a_{i}\right)\right|}{\lambda_{i}}+\sum_{i \neq j} \varepsilon_{i j}\right) .
$$

Subset 2: We consider the case of $u=\sum_{i=1}^{p} \alpha_{i} \tilde{\delta}_{i} \in V(p, \varepsilon)$ such that there exist $a_{i}$ satisfying $a_{i} \notin \bigcup_{y \in \mathcal{K}} B(y, \rho)$. We order the $\lambda_{i}$ 's in an increasing order, without loss of generality, we 
suppose that $\lambda_{1} \leq \cdots \leq \lambda_{p}$. Let $i_{1}$ be such that for any $i<i_{1}$, we have $a_{i} \in B\left(y_{l_{i}}, \rho\right), y_{l_{i}} \in \mathcal{K}$ and $a_{i_{1}} \notin \bigcup_{y \in \mathcal{K}} B(y, \rho)$. Let us define

$$
u_{1}=\sum_{i<i_{1}} \alpha_{i} \tilde{\delta}_{i}
$$

Observe that $u_{1}$ has to satisfy one of the three cases above, that is, $u \in V_{1}\left(i_{1}-1, \varepsilon\right)$ or $u_{1} \in V_{2}\left(i_{1}-1, \varepsilon\right)$ or $u_{1}$ satisfies the condition of Subset 1 . Thus we can apply the associated vector field, which we denote by $Y$, and we then have the following estimate:

$$
\langle\partial J(u), Y(u)\rangle \leq-c\left(\sum_{i<i_{1}} \frac{1}{\lambda_{i}^{\beta}}+\sum_{i<i_{1}} \frac{\left|\nabla H\left(a_{i}\right)\right|}{\lambda_{i}}+\sum_{i \neq j, i, j<i_{1}} \varepsilon_{i j}\right)+O\left(\sum_{i<i_{1}, j \geq i_{1}} \varepsilon_{i j}\right) .
$$

Now, we define the following vector field:

$$
Y^{\prime}=\frac{1}{\lambda_{i_{1}}} \frac{\partial \tilde{\delta}_{\left(a_{i_{1}} \lambda_{i_{1}}\right)}}{\partial a_{i_{1}}} \frac{\nabla K\left(a_{i_{1}}\right)}{\left|\nabla H\left(a_{i_{1}}\right)\right|}-c^{\prime} \sum_{i \geq i_{1}} 2^{i} Z_{i} .
$$

Using Proposition 2.6 and the fact that $\left|\nabla H\left(a_{i_{1}}\right)\right| \geq c>0$, we derive

$$
\left\langle\partial J(u), Y^{\prime}(u)\right\rangle \leq-\frac{c}{\lambda_{i_{1}}}+O\left(\sum_{i \neq i_{1}} \varepsilon_{i j}\right)-c^{\prime} \sum_{i \geq i_{1}, j \neq i} \varepsilon_{i j}+o\left(\sum_{i \geq i_{1}} \frac{1}{\lambda_{i}}\right) .
$$

Taking $c^{\prime}$ positive large enough, we find

$$
\left\langle\partial J(u), Y^{\prime}(u)\right\rangle \leq-c\left(\sum_{i=i_{1}}^{p} \frac{1}{\lambda_{i}^{\beta}}+\sum_{i=i_{1}}^{p} \frac{\left|\nabla H\left(a_{i}\right)\right|}{\lambda_{i}}+\sum_{i \geq i_{1}, j \neq i} \varepsilon_{i j}\right) .
$$

Now, let $W_{4}:=Y^{\prime}+m_{1} Y$, where $m_{1}$ is a small positive constant, we then have

$$
\left\langle\partial J(u), W_{4}(u)\right\rangle \leq-c\left(\sum_{i=1}^{p} \frac{1}{\lambda_{i}^{\beta}}+\sum_{i=1}^{p} \frac{\left|\nabla H\left(a_{i}\right)\right|}{\lambda_{i}}+\sum_{i \neq j} \varepsilon_{i j}\right) .
$$

Now, we define the pseudo-gradient $\widetilde{W}_{1}$ as a convex combination of $W_{i}$ for $i=1, \ldots, 4$. The construction of $\widetilde{W}_{1}$ is completed, it satisfies claim (i) of Theorem 3.2.

From the construction, $\widetilde{W}_{1}$ is bounded. Observe also that the only case where the maximum of the $\lambda_{i}$ 's increases is when $a_{i} \in B\left(y_{l_{i}}, \rho\right), y_{l_{i}} \in \mathcal{K} \forall i=1, \ldots, p$ with $\left(y_{l_{1}}, \ldots, y_{l_{p}}\right) \in \mathcal{C}_{n-2}^{+}$.

Now, arguing as in Appendix 2 of [26] (see also Appendix B of [6]), claim (ii) follows from (i) and the estimate of $\bar{v}$ given is ([22], Proposition 3.2). The proof of Theorem 3.2 is thereby completed.

Proof of Proposition 3.4 We divide the set $V_{2}(p, \varepsilon)$ into five sets.

$$
\begin{aligned}
V_{2}^{1}(p, \varepsilon)= & \left\{u=\sum_{i=1}^{p} \alpha_{i} \tilde{\delta}_{a_{i} \lambda_{i}} \in V_{2}(p, \varepsilon), y_{l_{i}} \neq y_{l_{j}} \forall i \neq j,-\sum_{k=1}^{n-1} b_{k}\left(y_{l_{i}}\right)>0,\right. \\
& \left.\lambda_{i}\left|a_{i}-y_{l_{i}}\right|<\delta \forall i=1, \ldots, p \text { and } \rho\left(y_{l_{i}}, \ldots, y_{l_{p}}\right)>0\right\},
\end{aligned}
$$




$$
\begin{aligned}
& V_{2}^{2}(p, \varepsilon)=\left\{u=\sum_{i=1}^{p} \alpha_{i} \tilde{\delta}_{a_{i} \lambda_{i}} \in V_{2}(p, \varepsilon), y_{l_{i}} \neq y_{l_{j}} \forall i \neq j,-\sum_{k=1}^{n-1} b_{k}\left(y_{l_{i}}\right)>0,\right. \\
& \left.\lambda_{i}\left|a_{i}-y_{l_{i}}\right|<\delta \forall i=1, \ldots, p \text { and } \rho\left(y_{l_{i}}, \ldots, y_{l_{p}}\right)<0\right\} \text {, } \\
& V_{2}^{3}(p, \varepsilon)=\left\{u=\sum_{i=1}^{p} \alpha_{i} \tilde{\delta}_{a_{i} \lambda_{i}} \in V_{2}(p, \varepsilon), y_{l_{i}} \neq y_{l_{j}} \forall i \neq j, \lambda_{i}\left|a_{i}-y_{l_{i}}\right|<\delta\right. \\
& \left.\forall i=1, \ldots, p \text {, and there exists } j \text { (at least) such that }-\sum_{k=1}^{n-1} b_{k}\left(y_{l_{j}}\right)<0\right\} \text {, } \\
& V_{2}^{4}(p, \varepsilon)=\left\{u=\sum_{i=1}^{p} \alpha_{i} \tilde{\delta}_{a_{i} \lambda_{i}} \in V_{2}(p, \varepsilon), y_{l_{i}} \neq y_{l_{j}} \forall i \neq j, \text { and there exists } j\right. \text { (at least) } \\
& \text { such that } \left.\lambda_{j}\left|a_{j}-y_{l_{j}}\right| \geq \frac{\delta}{2}\right\} \\
& V_{2}^{5}(p, \varepsilon)=\left\{u=\sum_{i=1}^{p} \alpha_{i} \tilde{\delta}_{a_{i} \lambda_{i}} \in V_{2}(p, \varepsilon) \text {, such that there exists } i \neq j\right. \text { satisfying } \\
& \left.y_{l_{i}}=y_{l_{j}}\right\} \text {. }
\end{aligned}
$$

We break up the proof into Steps 1-5 below. We construct an appropriate pseudo-gradient in each region and then glue up through convex combinations.

Step 1: First, we consider the case of $u=\sum_{i=1}^{p} \alpha_{i} \tilde{\delta}_{a_{i} \lambda_{i}} \in V_{2}^{1}(p, \varepsilon)$. We have, for any $i \neq j$, $\left|a_{i}-a_{j}\right|>\rho$, and therefore

$$
\begin{aligned}
\varepsilon_{i j} & =\left(\frac{2}{\left(1-\cos d\left(a_{i}, a_{j}\right)\right) \lambda_{i} \lambda_{j}}\right)^{\frac{n-2}{2}}(1+o(1)) \\
& =2^{\frac{n-2}{2}} \frac{G\left(a_{i}, a_{j}\right)}{\left(\lambda_{i} \lambda_{j}\right)^{\frac{n-2}{2}}}(1+o(1)),
\end{aligned}
$$

where $G\left(a_{i}, a_{j}\right)=\frac{1}{\left(1-\cos d\left(a_{i}, a_{j}\right)\right)^{\frac{n-2}{2}}}$, it is Green's function of $\left(S^{n}, \mathcal{P}\right)$. Thus,

$$
\lambda_{i} \frac{\partial \varepsilon_{i j}}{\partial \lambda_{i}}=-\frac{n-2}{2} 2^{\frac{n-2}{2}} \frac{G\left(a_{i}, a_{j}\right)}{\left(\lambda_{i} \lambda_{j}\right)^{\frac{n-2}{2}}}(1+o(1)) .
$$

Using Proposition 2.6 with $\beta=n-2$ and the fact that $\alpha_{i}^{\frac{4}{n-2}} H\left(a_{i}\right) J(u)^{\frac{n-1}{n-2}}=(1+o(1))$, we derive that

$$
\begin{aligned}
\left\langle\partial J(u), \alpha_{i} \lambda_{i} \frac{\partial \tilde{\delta}_{i}}{\partial \lambda_{i}}\right\rangle= & (n-2) J(u)^{1-\frac{n-1}{2}}\left[\frac{n-2}{n-1} \tilde{c}_{1} \frac{\sum_{i=1}^{p} b_{k}}{H\left(a_{i}\right)^{\frac{n}{4}}} \frac{1}{\lambda_{i}^{n-2}}\right. \\
& \left.+c_{1} 2^{\frac{n-4}{2}} \sum_{i \neq j} \frac{G\left(y_{l_{i}}, y_{l_{j}}\right)}{\left(H\left(a_{i}\right) H\left(a_{j}\right)\right)^{\frac{n-2}{4}}} \frac{1}{\left(\lambda_{i} \lambda_{j}\right)^{\frac{n-2}{2}}}\right] \\
& +o\left(\sum_{i=1}^{p} \frac{1}{\lambda_{i}^{n-2}}+\sum_{i \neq j} \varepsilon_{i j}\right),
\end{aligned}
$$


where $\tilde{c}_{1}=c_{0}^{\frac{2(n-1)}{n-2}} \int_{\mathbb{R}^{n-1}} \frac{\left|\left(x_{1}\right)\right|^{n-2}}{\left(1+|x|^{2}\right)^{n-1}} d x$. Hence, using the fact that $\left|a_{i}-y_{l_{i}}\right|<\delta, \delta$ very small, we get

$$
\begin{aligned}
\left\langle\partial J(u), \sum_{i=1}^{p} \alpha_{i} Z_{i}\right\rangle & \leq-c^{t} \Lambda M\left(y_{l_{1}}, \ldots, y_{l_{p}}\right) \Lambda+o\left(\sum_{i=1}^{p} \frac{1}{\lambda_{i}^{n-2}}+\sum_{i \neq j} \varepsilon_{i j}\right) \\
& \leq-c \rho\left(y_{l_{1}}, \ldots, y_{l_{p}}\right)|\Lambda|^{2}+o\left(\sum_{i=1}^{p} \frac{1}{\lambda_{i}^{n-2}}+\sum_{i \neq j} \varepsilon_{i j}\right),
\end{aligned}
$$

where $\Lambda={ }^{t}\left(\frac{1}{\lambda_{1}^{\frac{n-2}{2}}}, \ldots, \frac{1}{\lambda_{p}^{\frac{n-2}{2}}}\right)$. Here $M\left(y_{l_{1}}, \ldots, y_{l_{p}}\right)$ is defined in (1.1) and $\rho\left(y_{l_{1}}, \ldots, y_{l_{p}}\right)$ is the least eigenvalue of $M\left(y_{l_{1}}, \ldots, y_{l_{p}}\right)$. Using the fact that $\forall i \neq j$, we have $\varepsilon_{i j} \leq \frac{c}{\left(\lambda_{i} \lambda_{j}\right)^{\frac{n-2}{2}}}$. Since $\left|a_{i}-a_{j}\right| \geq \delta$, we then obtain

$$
\left\langle\partial J(u), \sum_{i=1}^{p} \alpha_{i} Z_{i}\right\rangle \leq-c\left(\sum_{i=1}^{p} \frac{1}{\lambda_{i}^{n-2}}+\sum_{i \neq j} \varepsilon_{i j}\right) .
$$

In addition, $\forall i=1, \ldots, p$, we have $\lambda_{i}\left|a_{i}\right|<\delta \Longrightarrow \frac{\left|\nabla H\left(a_{i}\right)\right|}{\lambda_{i}} \sim \frac{\mid\left(a_{i}\right)_{k} k^{\beta-1}}{\lambda_{i}} \leq \frac{c}{\lambda_{i}^{\beta}}$. Thus, we derive, for $W_{2}^{1}:=\sum_{i=1}^{p} \alpha_{i} Z_{i}$,

$$
\left\langle\partial J(u), W_{2}^{1}\right\rangle \leq-c\left(\sum_{i=1}^{p} \frac{1}{\lambda_{i}^{n-2}}+\sum_{i=1}^{p} \frac{\left|\nabla H\left(a_{i}\right)\right|}{\lambda_{i}}+\sum_{i \neq j} \varepsilon_{i j}\right) .
$$

Step 2: Secondly, we study the case of $u=\sum_{i=1}^{p} \alpha_{i} \tilde{\delta}_{a_{i} \lambda_{i}} \in V_{2}^{2}(p, \varepsilon)$. Let $e=\left(e_{i}\right)_{i=1, \ldots, p}$ be an eigenvector associated to $\rho\left(y_{l_{1}}, \ldots, y_{l_{p}}\right)$ such that $|e|=1$ with $e_{i}>0 \forall i=1, \ldots, p$. Let $\gamma>0$ be such that for any $x \in B(e, \gamma)=\left\{y \in S^{p-1}\right.$ s.t. $\left.|y-e| \leq \gamma\right\}$, we have

$$
{ }^{t} x M\left(y_{l_{1}}, \ldots, y_{l_{p}}\right) x \leq \frac{1}{2} \rho\left(y_{l_{1}}, \ldots, y_{l_{p}}\right) .
$$

\section{Two cases may occur.}

Case 1: $\frac{\Lambda}{|\Lambda|} \in B(e, \gamma)$, where $\Lambda={ }^{t}\left(\frac{1}{\lambda_{1}^{\frac{n-2}{2}}}, \ldots, \frac{1}{\lambda_{p}^{\frac{n-2}{2}}}\right)$. In this case, we define $W_{2}^{2}=-\sum_{i=1}^{p} \alpha_{i} Z_{i}$. As in Step 1, we find that

$$
\left\langle\partial J(u), W_{2}^{2}(u)\right\rangle \leq-c\left(\sum_{i=1}^{p} \frac{1}{\lambda_{i}^{\beta}}+\sum_{i=1}^{p} \frac{\left|\nabla H\left(a_{i}\right)\right|}{\lambda_{i}}+\sum_{i \neq j} \varepsilon_{i j}\right) .
$$

Case 2: $\frac{\Lambda}{|\Lambda|} \notin B(e, \gamma)$. In this case, we define

$$
W_{2}^{2}=-\frac{2}{n-2}|\Lambda| \sum_{i=1}^{p} \alpha_{i} \lambda_{i}^{\frac{n-1}{2}}\left[\frac{|\Lambda| e_{i}-\Lambda_{i}}{|\Lambda|}-\frac{\Lambda_{i}\langle|\Lambda| e-\Lambda, \Lambda\rangle}{|\Lambda|^{3}}\right] \frac{\partial \tilde{\delta}_{a_{i} \lambda_{i}}}{\partial \lambda_{i}}
$$

Using Proposition 2.6, we find that

$$
\left\langle\partial J(u), W_{2}^{2}(u)\right\rangle=-c|\Lambda|^{2} \frac{\partial}{\partial t}\left({ }^{t} \Lambda(t) M \Lambda(t)\right)_{\left.\right|_{t=0}}+o\left(\sum_{i=1}^{p} \frac{1}{\lambda_{i}^{n-2}}\right)+o\left(\sum_{i \neq j} \varepsilon_{i j}\right),
$$


where $M=M\left(y_{l_{1}}, \ldots, y_{l_{p}}\right)$ and $\Lambda(t)=\frac{(1-t) \Lambda+t|\Lambda| e}{|(1-t) \Lambda+t| \Lambda|e|} \Lambda$. Observe that

$$
{ }^{t} \Lambda(t) M \Lambda(t)=\rho+\frac{(1-t)^{2}}{|(1-t) \Lambda+t| \Lambda|e|}\left({ }^{t} \Lambda M \Lambda-\rho|\Lambda|^{2}\right) .
$$

Thus we obtain $\frac{\partial}{\partial t}\left({ }^{t} \Lambda(t) M \Lambda(t)\right)<-c$, and therefore we get

$$
\left\langle\partial J(u), W_{2}^{2}(u)\right\rangle \leq-c\left(\sum_{i=1}^{p} \frac{1}{\lambda_{i}^{n-4}}+\sum_{i=1}^{p} \frac{\left|\nabla H\left(a_{i}\right)\right|}{\lambda_{i}}+\sum_{i \neq j} \varepsilon_{i j}\right) .
$$

Step 3: Now, we deal with the case of $u=\sum_{i=1}^{p} \alpha_{i} \tilde{\delta}_{a_{i} \lambda_{i}} \in V_{2}^{3}(p, \varepsilon)$.

Without loss of generality, we can assume that $1, \ldots, q$ are the indices which satisfy $-\sum_{k=1}^{n-1} b_{k}\left(y_{l_{i}}\right)<0 \forall i=1, \ldots, q$. Let

$$
\widetilde{W}_{2}^{1}=\sum_{i=1}^{q}-\alpha_{i} Z_{i}
$$

By Proposition 2.6 and (3.8), we obtain

$$
\left\langle\partial J(u), \widetilde{W}_{2}^{1}(u)\right\rangle \leq-c\left(\sum_{i=1}^{q} \frac{1}{\lambda_{i}^{\beta}}+\sum_{i \neq j, 1 \leq i \leq q} \varepsilon_{i j}\right) .
$$

Set

$$
I=\left\{i, 1 \leq i \leq p \text { s.t. } \lambda_{i} \leq \frac{1}{10} \min _{1 \leq j \leq q} \lambda_{j}\right\}
$$

It is easy to see that we can add to the above estimates all indices $i$ such that $i \notin I$. Thus

$$
\left\langle\partial J(u), \widetilde{W}_{2}^{1}(u)\right\rangle \leq-c\left(\sum_{i \notin I} \frac{1}{\lambda_{i}^{\beta}}+\sum_{i \neq j, i \notin I} \varepsilon_{i j}\right) .
$$

If $I \neq \varnothing$, in this case, we write $u$ as follows:

$$
u=\sum_{i \in I} \alpha_{i} \tilde{\delta}_{a_{i} \lambda_{i}}+\sum_{i \notin I} \alpha_{i} \tilde{\delta}_{a_{i} \lambda_{i}}=u_{1}+u_{2}
$$

Observe that $u_{1}$ has to satisfy one of the two cases above, that is, $u_{1} \in V_{2}^{1}(\sharp I, \varepsilon)$ or $u_{1} \in$ $V_{2}^{2}(\sharp I, \varepsilon)$. Thus we can apply the associated vector field, which we denote by $\widetilde{W}_{2}^{2}$. We then have

$$
\left\langle\partial J(u), \widetilde{W}_{2}^{2}(u)\right\rangle \leq-c\left(\sum_{i \in I} \frac{1}{\lambda_{i}^{\beta}}+\sum_{i \neq j, i \in I} \varepsilon_{i j}+\sum_{i=1}^{p} \frac{\left|\nabla H\left(a_{i}\right)\right|}{\lambda_{i}}\right)+O\left(\sum_{i \neq j, i \notin I} \varepsilon_{i j}\right) .
$$

Let, in this subset $W_{2}^{3}=\widetilde{W}_{2}^{1}+m_{1} \widetilde{W}_{2}^{2}, m_{1}$ be a small positive constant. We get

$$
\left\langle\partial J(u), W_{2}^{3}(u)\right\rangle \leq-c\left(\sum_{i=1}^{p} \frac{1}{\lambda_{i}^{\beta}}+\sum_{i=1}^{p} \frac{\left|\nabla H\left(a_{i}\right)\right|}{\lambda_{i}}+\sum_{i \neq j} \varepsilon_{i j}\right) .
$$


Step 4: We consider here the case of $u=\sum_{i=1}^{p} \alpha_{i} \tilde{\delta}_{a_{i} \lambda_{i}} \in V_{2}^{4}(p, \varepsilon)$.

We order the $\lambda_{i}$ 's in an increasing order. For the sake of simplicity, we can assume that $\lambda_{1} \leq \cdots \leq \lambda_{p}$. Let $\lambda_{i_{1}}=\inf \left\{\lambda_{j}\right.$ s.t. $\left.\lambda_{j}\left|a_{j}\right| \geq \delta\right\}$. For $m_{1}>0$ small enough, we need to prove the following claim:

$$
\left\langle\partial J(u),\left(X_{i_{1}}-m_{1} Z_{i_{1}}\right)(u)\right\rangle \leq-c\left(\sum_{i=i_{1}}^{p} \frac{1}{\lambda_{i}^{\beta}}+\sum_{j \neq i_{1}} \varepsilon_{i_{1} j}+\sum_{i=1}^{p} \frac{\left|\nabla H\left(a_{i_{1}}\right)\right|}{\lambda_{i_{1}}}\right) .
$$

Indeed, for $i \neq j$, we have $\left|a_{i}-a_{j}\right|>\rho$. Thus in Proposition 2.6 the term $\left|\frac{1}{\lambda_{i}} \frac{\partial \varepsilon_{i j}}{\partial\left(a_{i}\right)_{k}}\right|$ is very small with respect to $\varepsilon_{i j}$. Hence,

$$
\begin{aligned}
\left\langle\partial J(u), X_{i_{1}}(u)\right\rangle \leq & -\frac{c}{\lambda_{i_{1}}^{\beta}}\left(\int_{\mathbb{R}^{n-1}} b_{k_{i_{1}}} \frac{\left|x_{k_{i_{1}}}+\lambda_{i_{1}}\left(a_{i_{1}}\right)_{k_{i_{1}}}\right|^{\beta}}{\left(1+\lambda_{i_{1}}\left|\left(a_{i_{1}}\right)_{k_{i_{1}}}\right|\right)^{\frac{\beta-1}{2}}} \frac{x_{k_{i_{1}}}}{\left(1+|x|^{2}\right)^{n}} d x\right)^{2} \\
& +o\left(\frac{1}{\lambda_{i_{1}}^{\beta}}\right)+o\left(\sum_{j \neq i_{1}} \varepsilon_{i_{1} j}\right) .
\end{aligned}
$$

If $i_{1} \in L_{1}$ in this case $\delta \leq \lambda_{i_{1}}\left|a_{i_{1}}\right| \leq M_{1}$, using (3.18), we get

$$
\begin{aligned}
\left\langle\partial J(u), X_{i_{1}}(u)\right\rangle & \leq-c \frac{1}{\lambda_{i_{1}}^{\beta}}+o\left(\sum_{j \neq i_{1}} \varepsilon_{i_{1} j}\right) \\
& \leq-c \sum_{i=i_{1}}^{p} \frac{1}{\lambda_{i}^{\beta}}+o\left(\sum_{j \neq i_{1}} \varepsilon_{i_{1} j}\right) .
\end{aligned}
$$

From another part, we have by Proposition 2.6 and (3.6)

$$
\left\langle\partial J(u), Z_{i_{1}}(u)\right\rangle \leq-c \sum_{j \neq i_{1}} \varepsilon_{i_{1} j}+O\left(\frac{1}{\lambda_{i_{1}}^{\beta}}\right) .
$$

Using (3.21) and (3.22), our claim follows in this case.

If $i_{1} \in L_{2}$, using (3.3), we find

$$
\begin{aligned}
\left\langle\partial J(u), X_{i_{1}}(u)\right\rangle & \leq-c\left(\frac{1}{\lambda_{i_{1}}^{\beta}}+\frac{\left|\left(a_{i_{1}}\right)_{k_{i_{1}}}\right|^{\beta-1}}{\lambda_{i_{1}}}\right)+o\left(\sum_{j \neq i_{1}} \varepsilon_{i_{1} j}\right) \\
& \leq-c\left(\sum_{i=i_{1}}^{p} \frac{1}{\lambda_{i}^{\beta}}+\frac{\left|\left(a_{i_{1}}\right)_{k_{i_{1}}}\right|^{\beta-1}}{\lambda_{i_{1}}}\right)+o\left(\sum_{j \neq i_{1}} \varepsilon_{i_{1} j}\right),
\end{aligned}
$$

and by Proposition 2.6 and (3.6), we have

$$
\left\langle\partial J(u),-Z_{i_{1}}(u)\right\rangle \leq-c \sum_{j \neq i_{1}} \varepsilon_{i_{1} j}+O\left(\frac{\left|\left(a_{i_{1}}\right)_{k_{i_{1}}}\right|^{\beta-2}}{\lambda_{i_{1}}^{2}}\right) .
$$


Now, using (3.9), we obtain

$$
\begin{aligned}
\left\langle\partial J(u),\left(X_{i_{1}}-m_{1} Z_{i_{1}}\right)(u)\right\rangle & \leq-c\left(\sum_{i=i_{1}}^{p} \frac{1}{\lambda_{i}^{\beta}}+\sum_{j \neq i_{1}} \varepsilon_{i_{1} j}+\frac{\left|\left(a_{i_{1}}\right)_{k}\right|^{\beta-1}}{\lambda_{i_{1}}}\right) \\
& \leq-c\left(\sum_{i=i_{1}}^{p} \frac{1}{\lambda_{i}^{\beta}}+\sum_{j \neq i_{1}} \varepsilon_{i_{1} j}+\frac{\left|\nabla H\left(a_{i_{1}}\right)\right|}{\lambda_{i_{1}}}\right),
\end{aligned}
$$

since $\left|\nabla H\left(a_{i_{1}}\right)\right| \sim\left|\left(a_{i_{1}}\right)_{k_{i}}\right|^{\beta-1}$, hence our claim is valid.

Now, let

$$
I=\left\{i, 1 \leq i \leq p \text { s.t. } \lambda_{i}<\frac{1}{10} \lambda_{i_{1}}\right\}
$$

it is easy to see that

$$
\left\langle\partial J(u),\left(X_{i_{1}}-m_{1} Z_{i_{1}}\right)(u)\right\rangle \leq-c\left(\sum_{i \notin I} \frac{1}{\lambda_{i}^{\beta}}+\sum_{j \neq i, i \notin I} \varepsilon_{i j}+\frac{\left|\nabla H\left(a_{i_{1}}\right)\right|}{\lambda_{i_{1}}}\right)
$$

since $\varepsilon_{i j} \leq \frac{c}{\left(\lambda_{i} \lambda_{j}\right)^{\frac{n-2}{2}}}$. Furthermore, using (3.3), we have

$$
\left\langle\partial J(u),\left(X_{i_{1}}-m_{1} Z_{i_{1}}+\sum_{i \notin I, i \in L_{2}} X_{i}\right)(u)\right\rangle \leq-c\left(\sum_{i \notin I} \frac{1}{\lambda_{i}^{\beta}}+\sum_{i \notin I} \frac{\left|\nabla H\left(a_{i}\right)\right|}{\lambda_{i}}+\sum_{i \neq j, i \notin I} \varepsilon_{i j}\right)
$$

since, for $i \notin I$ and $i \in L_{1}$, we have $\frac{\left|\nabla H\left(a_{i}\right)\right|}{\lambda_{i}} \leq \frac{c}{\lambda_{i}^{\beta}}$.

We need to add the remaining terms (if $I \neq \varnothing$ ). Let $u_{1}=\sum_{i \in I} \alpha_{i} \tilde{\delta}_{a_{i} \lambda_{i}} \forall i \in I$, we have $\lambda_{i}\left|a_{i}\right|<\delta$, thus $u_{1} \in V_{2}^{j}(\sharp I, \varepsilon), j=1$ or 2 or 3 . We can apply then the associated vector field, which we denote by $\widetilde{W}_{2}^{4}$. We then have

$$
\left\langle\partial J(u), \widetilde{W}_{2}^{4}\right\rangle \leq-c\left(\sum_{i \in I} \frac{1}{\lambda_{i}^{\beta}}+\sum_{i \neq j, i, j \in I} \varepsilon_{i j}+\sum_{i \in I} \frac{\left|\nabla H\left(a_{i}\right)\right|}{\lambda_{i}}\right)+O\left(\sum_{i \in I, j \notin I} \varepsilon_{i j}\right) .
$$

Let $W_{2}^{4}=X_{i_{1}}-m_{1} Z_{i_{1}}+\sum_{i \notin I, i \in L_{2}} X_{i}+m_{2} \widetilde{W}_{2}^{4}, m_{2}$ is positive small enough, we get

$$
\left\langle\partial J(u), W_{2}^{4}(u)\right\rangle \leq-c\left(\sum_{i=1}^{p} \frac{1}{\lambda_{i}^{\beta}}+\sum_{i=1}^{p} \frac{\left|\nabla H\left(a_{i}\right)\right|}{\lambda_{i}}+\sum_{i \neq j} \varepsilon_{i j}\right) .
$$

Step 5: We study now the case of $u=\sum_{i=1}^{p} \alpha_{i} \tilde{\delta}_{a_{i} \lambda_{i}} \in V_{2}^{5}(p, \varepsilon)$.

Let

$$
B_{k}=\left\{j, 1 \leq j \leq p \text { s.t. } a_{j} \in B\left(y_{l_{k}}, \rho\right)\right\}
$$

In this case, there is at least one $B_{k}$ which contains at least two indices. Without loss of generality, we can assume that $1, \ldots, q$ are the indices such that the set $B_{k}, 1 \leq k \leq q$, contains at least two indices. We decrease the $\lambda_{i}$ 's for $i \in B_{k}$ with different speed. For this purpose, 
let

$$
\begin{aligned}
& \chi: \mathbb{R} \rightarrow \mathbb{R}^{+}, \\
& t \longmapsto \begin{cases}0 & \text { if }|t| \leq \gamma^{\prime}, \\
1 & \text { if }|t| \geq 1,\end{cases}
\end{aligned}
$$

where $\gamma^{\prime}$ is a small constant.

$$
\begin{aligned}
& \text { For } j \in B_{k} \text {, set } \bar{\chi}\left(\lambda_{j}\right)=\sum_{i \neq j, i \in B_{k}} \chi\left(\frac{\lambda_{j}}{\lambda_{i}}\right) \text {. Define } \\
& \qquad \widetilde{W}_{2}^{5}=-\sum_{k=1}^{q} \sum_{j \in B_{k}} \alpha_{j} \bar{\chi}\left(\lambda_{j}\right) Z_{j} .
\end{aligned}
$$

Using Proposition 2.6 and (3.6), we obtain

$$
\begin{aligned}
\left\langle\partial J(u), \widetilde{W}_{2}^{5}(u)\right\rangle \leq & c \sum_{k=1}^{q}\left[\sum_{i \neq j ; j, j \in B_{k}} \bar{\chi}\left(\lambda_{j}\right) \lambda_{j} \frac{\partial \varepsilon_{i j}}{\partial \lambda_{j}}+\sum_{j \in B_{k}, j \in L_{1}} \bar{\chi}\left(\lambda_{j}\right) O\left(\frac{1}{\lambda_{j}^{\beta}}\right)\right. \\
& \left.+\sum_{j \in B_{k}, j \in L_{2}} \bar{\chi}\left(\lambda_{j}\right) O\left(\frac{\left|\left(a_{j}\right)_{k_{i}}\right|^{\beta-2}}{\lambda_{j}^{2}}\right)\right] .
\end{aligned}
$$

For $j \in B_{k}$, with $k \leq q$, if $\bar{\chi}\left(\lambda_{j}\right) \neq 0$, then there exists $i \in B_{k}$ such that $\frac{1}{\lambda_{j}^{\beta}}=o\left(\varepsilon_{i j}\right)$ (for $\rho$ small enough). Furthermore, for $j \in B_{k}$, if $i \notin B_{k}$ (or $i \in B_{k}$ with $\lambda_{i} \sim \lambda_{j}$ ), then we have by (3.8)

$$
\lambda_{j} \frac{\partial \varepsilon_{i j}}{\partial \lambda_{j}} \leq-c \varepsilon_{i j} \quad \text { and } \quad \lambda_{i} \frac{\partial \varepsilon_{i j}}{\partial \lambda_{i}} \leq-c \varepsilon_{i j}
$$

In the case where $i \in B_{k}$ with (assuming $\lambda_{i} \ll \lambda_{j}$ ), we have $\bar{\chi}\left(\lambda_{j}\right)-\bar{\chi}\left(\lambda_{i}\right) \geq 1$. Thus

$$
\bar{\chi}\left(\lambda_{j}\right) \lambda_{j} \frac{\partial \varepsilon_{i j}}{\partial \lambda_{j}}+\bar{\chi}\left(\lambda_{i}\right) \lambda_{i} \frac{\partial \varepsilon_{i j}}{\partial \lambda_{i}} \leq \lambda_{j} \frac{\partial \varepsilon_{i j}}{\partial \lambda_{j}} \leq-c \varepsilon_{i j}
$$

Thus we obtain

$$
\begin{aligned}
\left\langle\partial J(u), \widetilde{W}_{2}^{5}(u)\right\rangle \leq & -c \sum_{k=1}^{q} \sum_{j \in B_{k}} \bar{\chi}\left(\lambda_{j}\right)\left(\sum_{i \neq j} \varepsilon_{i j}+\frac{1}{\lambda_{j}^{\beta}}\right) \\
& +\sum_{k=1}^{q} \sum_{j \in B_{k}, j \in L_{2}} \bar{\chi}\left(\lambda_{j}\right) O\left(\frac{\left|\left(a_{j}\right)_{k_{i}}\right|^{\beta-2}}{\lambda_{j}^{2}}\right) .
\end{aligned}
$$

We need to add the indices $j, j \in{ }^{C}\left(\bigcup_{K=1}^{q} B_{k}\right) \cup\left\{j \in B_{k}\right.$ s.t. $\left.\bar{\chi}\left(\lambda_{j}\right)=0\right\}$. Let

$$
\lambda_{i_{0}}=\inf \left\{\lambda_{i}, i=1, \ldots, p\right\} .
$$

We distinguish two cases. 
Case 1: There exists $j$ such that $\bar{\chi}\left(\lambda_{j}\right) \neq 0$ and $\lambda_{i_{0}} \sim \lambda_{j}\left(\gamma^{\prime} \leq \frac{\lambda_{i_{0}}}{\lambda_{j}} \leq 1\right)$, then we can make appear $-\frac{1}{\lambda_{i_{0}}^{\beta}}$ in the above estimate, and therefore $-\sum_{i=1}^{p} \frac{1}{\lambda_{i}^{\beta}}$ and $-\sum_{k \neq r} \varepsilon_{k r}$. Thus we obtain

$$
\left\langle\partial J(u), \widetilde{W}_{2}^{5}(u)\right\rangle \leq-c\left(\sum_{i=1}^{p} \frac{1}{\lambda_{i}^{\beta}}+\sum_{i \neq j} \varepsilon_{i j}\right)+O\left(\sum_{k=1}^{q} \sum_{j \in B_{k}, j \in L_{2}} \frac{\left|\left(a_{j}\right)_{k_{i}}\right|^{\beta-2}}{\lambda_{j}^{2}}\right) .
$$

Now, let

$$
W_{2}^{5}=\widetilde{W}_{2}^{5}+m_{1} \sum_{i=1}^{p} X_{i}
$$

Using the above estimates with Proposition 2.6 and (3.9), we obtain

$$
\left\langle\partial J(u), W_{2}^{5}(u)\right\rangle \leq-c\left(\sum_{i=1}^{p} \frac{1}{\lambda_{i}^{\beta}}+\sum_{i \neq j} \varepsilon_{i j}+\sum_{i=1}^{p} \frac{\left|\nabla H\left(a_{i}\right)\right|}{\lambda_{i}}\right) .
$$

Case 2: For each $j \in B_{k}, 1 \leq k \leq q$, we have

$$
\lambda_{i_{0}} \ll \lambda_{j}\left(i . e ., \frac{\lambda_{i_{0}}}{\lambda_{j}}<\gamma^{\prime}\right) \quad \text { or if } \quad \lambda_{i_{0}} \sim \lambda_{j}, \quad \text { we have } \bar{\chi}\left(\lambda_{j}\right)=0 \text {. }
$$

In this case, we define

$$
D=\left[\left\{i, \bar{\chi}\left(\lambda_{i}\right)=0\right\} \cup^{C}\left(\bigcup_{k=1}^{q} B_{k}\right)\right] \cap\left\{i, \frac{\lambda_{i}}{\lambda_{i_{0}}}<\frac{1}{\gamma^{\prime}}\right\} .
$$

It is easy to see that $i_{0} \in D$ and if $i \neq j \in\left\{i, \bar{\chi}\left(\lambda_{i}\right)=0\right\} \cup{ }^{C}\left(\bigcup_{k=1}^{q} B_{k}\right)$, we have $a_{i} \in B\left(y_{l_{i}}, \rho\right)$ and $a_{j} \in B\left(y_{l_{j}}, \rho\right)$ with $y_{l_{i}} \neq y_{l_{j}}$. Let

$$
u_{1}=\sum_{i \in D} \alpha_{i} \tilde{\delta}_{a_{i} \lambda_{i}}
$$

$u_{1}$ has to satisfy one of the four subsets above, that is, $u_{1} \in V_{2}^{j}(\sharp I, \varepsilon)$ for $j=1,2,3$ or 4 . Thus we can apply the associated vector field, which we denote by $Y$, and we have the estimate

$$
\langle\partial J(u), Y(u)\rangle \leq-c\left(\sum_{i \in D} \frac{1}{\lambda_{i}^{\beta}}+\sum_{i \in D} \frac{\left|\nabla H\left(a_{i}\right)\right|}{\lambda_{i}}+\sum_{i \neq j, i, j \in D} \varepsilon_{i j}\right)+O\left(\sum_{i \in D, j \notin D} \varepsilon_{i j}\right) .
$$

Observe that in the above majorization we have the term $-\frac{1}{\lambda_{i_{0}}^{\beta}}$, thus we can make appear $-\sum_{i=1}^{p} \frac{1}{\lambda_{i}^{\beta}}$. Now, concerning the term $-\sum_{i \neq j} \varepsilon_{i j}$, if $i \in D$ and $j \in{ }^{C} D$, observe that

$$
{ }^{C} D=\left\{i, \frac{\lambda_{i}}{\lambda_{i_{0}}}>\frac{1}{\gamma^{\prime}}\right\} \cup\left[\left\{i, \bar{\chi}\left(\lambda_{i}\right) \neq 0\right\} \cap\left(\bigcup_{k=1}^{q} B_{k}\right)\right]
$$


We have two situations: either $j \in\left[\left\{i, \bar{\chi}\left(\lambda_{i}\right) \neq 0\right\} \cap\left(\bigcup_{k=1}^{q} B_{k}\right)\right]$, then we have $-\varepsilon_{i j}$ in the estimates (3.23), or $j \in\left\{i, \frac{\lambda_{i}}{\lambda_{i_{0}}}>\frac{1}{\gamma^{\prime}}\right\}$. We can prove in these cases that $\left|a_{i}-a_{j}\right| \geq \rho$. Thus

$$
\varepsilon_{i j} \leq \frac{c}{\left(\lambda_{i} \lambda_{j}\right)^{\frac{n-2}{2}}}<\frac{c \gamma^{\prime \frac{n-2}{2}}}{\left(\lambda_{i_{0}} \lambda_{i}\right)^{\frac{n-2}{2}}}=o\left(\varepsilon_{i_{0} i}\right) \quad\left(\text { for } \gamma^{\prime} \text { small enough }\right)
$$

Thus we derive

$$
\begin{aligned}
\left\langle\partial J(u),\left(\widetilde{W}_{2}^{5}+m_{1} Y\right)(u)\right\rangle \leq & -c\left(\sum_{i \in D} \frac{\left|\nabla H\left(a_{i}\right)\right|}{\lambda_{i}}+\sum_{i=1}^{p} \frac{1}{\lambda_{i}^{\beta}}+\sum_{i \neq j} \varepsilon_{i j}\right) \\
& +\sum_{K=1}^{q} \sum_{j \in B_{k}, j \in L_{2}} \bar{\chi}\left(\lambda_{j}\right) O\left(\frac{\left|\left(a_{j}\right)_{k_{i}}\right|^{\beta-2}}{\lambda_{j}^{2}}\right),
\end{aligned}
$$

and hence, by (3.9), we have

$$
\left\langle\partial J(u),\left(\widetilde{W}_{2}^{5}+m_{1} Y+m_{2} \sum_{i=1, i \in L_{2}} X_{i}\right)(u)\right\rangle \leq-c\left(\sum_{i=1}^{p} \frac{1}{\lambda_{i}^{\beta}}+\sum_{i \neq j} \varepsilon_{i j}+\sum_{i=1}^{p} \frac{\left|\nabla H\left(a_{i}\right)\right|}{\lambda_{i}}\right)
$$

for $m_{1}$ and $m_{2}$ two small positive constants. In this case, we define

$$
W_{2}^{5}:=\widetilde{W}_{2}^{5}+m_{1} Y+m_{2} \sum_{i=1, i \in L_{2}} X_{i}
$$

The vector field $W_{2}$ in $V_{2}(p, \varepsilon)$ is a convex combination of $W_{2}^{j}, j=1, \ldots, 5$. This concludes the proof of Proposition 3.4.

Corollary 3.5 Let $H$ be a $C^{2}$-function on $\partial \mathbb{B}^{n}$ satisfying $(\mathfrak{f})_{\beta}$ condition with $n-2 \leq \beta \leq$ $n-1$. The only critical points at infinity of $J$ in $V(p, \varepsilon), p \geq 1$ are

$$
\sum_{i=1}^{p} \alpha_{i} \tilde{\delta}_{\left(y_{i}, \infty\right)}, \quad \text { where }\left(y_{1}, \ldots, y_{p}\right) \in C_{n-2}^{+} \cup\left(\mathcal{K}^{+} \backslash \mathcal{K}_{n-2}\right)
$$

The Morse index of such a critical point at infinity is

$$
i\left(y_{1}, \ldots, y_{p}\right)=p-1+\sum_{j=1}^{p}(n-1)-\tilde{i}\left(y_{j}\right) .
$$

\section{Proof of the result}

Proof of Theorem 1.1 We prove the existence result by contradiction. Therefore, we assume that equation (1.1) has no solution. It follows from Corollary 3.5 that the critical points at infinity of the associated variational problem are in one-to-one correspondence with the elements of $C_{n-2}^{+} \cup\left(\mathcal{K}^{+} \backslash \mathcal{K}_{n-2}\right)$.

Notice that, just like for usual critical points, it is associated to each critical point at infinity $w_{\infty}$ of $J$ stable and unstable manifolds $W_{s}^{\infty}\left(w_{\infty}\right)$ and $W_{u}^{\infty}\left(w_{\infty}\right)$ (see [26], pp.356357). These manifolds can be easily described once a finite dimensional reduction, like the one we performed in Section 3, is established. 
For any $w_{\infty}=\left(y_{i_{1}}, \ldots, y_{i_{p}}\right) \in C_{n-2}^{+}$, let $c(w)_{\infty}=S_{n}\left(\sum_{j=1}^{p} \frac{1}{K\left(y_{i_{j}}\right)^{\frac{n-2}{2}}}\right)^{\frac{2}{n}}$ denote the associated critical value. Here we choose to consider a simplified situation, where for any $w_{\infty} \neq w_{\infty}^{\prime}$, $c(w)_{\infty} \neq c\left(w^{\prime}\right)_{\infty}$, and thus order the $c(w)_{\infty}$ 's, $w_{\infty} \in C_{n-2}^{+}$as

$$
c\left(w_{1}\right)_{\infty}<\cdots<c\left(w_{k_{0}}\right)_{\infty}
$$

By using a deformation lemma (see Proposition 7.24 and Theorem 8.2 of [28]), we know that if $c\left(w_{k-1}\right)_{\infty}<a<c\left(w_{k}\right)_{\infty}<b<c\left(w_{k+1}\right)_{\infty}$, then

$$
J_{b} \simeq J_{a} \cup W_{u}^{\infty}\left(w_{k}\right)_{\infty}
$$

where $J_{b}=\left\{u \in \Sigma^{+}, J(u) \leq b\right\}$ and $\simeq$ denotes retracts by deformation.

We apply the Euler-Poincaré characteristic to both sides of (4.1), and we find that

$$
\chi\left(J_{b}\right)=\chi\left(J_{a}\right)+(-1)^{i\left(w_{k}\right)_{\infty}},
$$

where $i\left(w_{k}\right)_{\infty}$ denotes the index of the critical point at infinity $\left(w_{k}\right)_{\infty}$. Let

$$
b_{1}<c\left(w_{1}\right)_{\infty}=\min _{u \in \Sigma^{+}} J(u)<b_{2}<c\left(w_{2}\right)_{\infty}<\cdots<b_{k_{0}}<c\left(w_{k_{0}}\right)_{\infty}<b_{k_{0}+1} .
$$

Since we have assumed that (1.1) has no solution, $J_{b_{k_{0}+1}}$ is a retard by deformation of $\Sigma^{+}$. Therefore $\chi\left(b_{k_{k_{0}+1}}\right)=1$ since $\Sigma^{+}$is a contractible set. Now, using (4.2), we derive, after recalling that $\chi\left(J_{b_{1}}\right)=\chi(\varnothing)=0$,

$$
1=\sum_{j=1}^{k_{0}}(-1)^{i\left(w_{j}\right)_{\infty}} .
$$

Hence, if (4.3) is violated, (1.1) has a solution.

To prove the multiplicity part of the statement, we observe that it follows from the SardSmale theorem that for generic $H$ 's, the solutions of (1.1) are all non-degenerate in the sense that the associated linearized operator does not admit zero as an eigenvalue. We need to introduce the following lemma extracted from [9].

Lemma 4.1 (see [22], Section 3.2) Let $w$ be a solution of (1.1). Assume that the function $H$ satisfies condition $(\mathfrak{f})_{\beta}$, with $\frac{n-2}{2}<\beta \leq n-2$. Then, for each $p \in \mathbb{N}^{*}$, there is no critical points neither critical points at infinity in $V(p, \varepsilon, w)$.

Once the existence of mixed critical points at infinity is ruled out, it follows from the above arguments that

$$
\Sigma^{+} \simeq \bigcup_{j=1}^{k_{0}} W_{u}^{\infty}\left(w_{j}\right)_{\infty} \cup \bigcup_{w, \partial J(w)=0} W_{u}(w) .
$$

Now using the Euler-Poincaré theorem, we derive that

$$
1=\sum_{j=1}^{k_{0}}(-1)^{i\left(w_{j}\right)_{\infty}}+\sum_{w, \partial J(w)=0}(-1)^{\operatorname{morse}(w)} .
$$

Hence our theorem follows. 


\section{Competing interests}

The authors declare that they have no competing interests.

\section{Authors' contributions}

The authors did not provide this information.

Received: 16 December 2012 Accepted: 12 July 2013 Published: 22 August 2013

\section{References}

1. Li, YY: The Nirenberg problem in a domain with boundary. Topol. Methods Nonlinear Anal. 6, 309-329 (1995)

2. Aubin, T, Bahri, A: Méthodes de topologie algébrique pour le problème de la courbure scalaire prescrite (French) (Methods of algebraic topology for the problem of prescribed scalar curvature). J. Math. Pures Appl. 76(6), 525-549 (1997)

3. Aubin, T, Bahri, A: Une hypothèse topologique pour le problème de la courbure scalaire prescrite (French) (A topological hypothesis for the problem of prescribed scalar curvature). J. Math. Pures Appl. 76(10), 843-850 (1997)

4. Bahri, A, Coron, JM: The scalar curvature problem on the standard three dimensional spheres. J. Funct. Anal. 95, 106-172 (1991)

5. Bahri, A, Coron, JM: On a nonlinear elliptic equation involving the critical Sobolev exponent: the effect of topology of the domain. Commun. Pure Appl. Math. 41, 255-294 (1988)

6. Ben Ayed, M, Chen, Y, Chtioui, H, Hammami, M: On the prescribed scalar curvature problem on 4-manifolds. Duke Math. J. 84, 633-677 (1996)

7. Ben Mahmoud, R, Chtioui, $\mathrm{H}$ : Existence results for the prescribed scalar curvature on $\mathbb{S}^{3}$. Ann. Inst. Fourier $61,971-986$ (2011)

8. Chtioui, H: Prescribing the scalar curvature problem on three and four manifolds. Adv. Nonlinear Stud. 3, 457-470 (2003)

9. Chtioui, H, Rigane, A: On the prescribed Q-curvature problem on $S^{n}$. J. Funct. Anal. 261, 2999-3043 (2011)

10. Escobar, JF: Conformal deformation of Riemannian metric to scalar flat metric with constant mean curvature on the boundary. Ann. Math. 2(136), 1-50 (1992)

11. Escobar, JF: Conformal metric with prescribed mean curvature on the boundary. Calc. Var. Partial Differ. Equ. 4, 559-592 (1996)

12. Escobar, JF: The Yamabe problem on manifolds with boundary. J. Differ. Geom. 35, 21-84 (1992)

13. Felli, V, Ould Ahmedou, M: Compactness result in conformal deformations on manifolds with boundary. Math. Z. 244 175-210 (2003)

14. Felli, V, Ould Ahmedou, M: On a geometric equation with critical nonlinearity on the boundary. Pac. J. Math. 219, 1-25 (2005)

15. Schoen, R: On the number of solutions of constant scalar curvature in a conformal class. In: Lawson, HB, Tenenblat, $\mathrm{K}$ (eds.) Differential Geometry: A Symposium in Honor of Manfredo Do Carmo, pp. 311-320. Wiley, New York (1991)

16. Schoen, R, Zhang, D: Prescribed scalar curvature on the $n$-sphere. Calc. Var. Partial Differ. Equ. 4, 1-25 (1996)

17. Escobar, JF, Garcia, G: Conformal metric on the ball with zero scalar and prescribed mean curvature on the boundary. J. Funct. Anal. 211(1), 71-152 (2004)

18. Abdelhedi, W, Chtioui, H, Ould Ahmedou, M: Conformal metrics with prescribed boundary mean curvature on balls. Ann. Glob. Anal. Geom. 36(4), 327-362 (2009)

19. Abdelhedi, W, Chtioui, $\mathrm{H}$ : The prescribed boundary mean curvature problem on standard $n$-dimensional ball. Nonlinear Anal. TMA 67, 668-686 (2007)

20. Abdelhedi, W, Chtioui, H, Ould Ahmedou, M: A Morse theoretical approach for boundary mean curvature problem on $B^{4}$. J. Funct. Anal. 254, 1307-1341 (2008)

21. Chang, SA, Xu, X, Yang, P: A perturbation result for prescribing mean curvature. Math. Ann. 310(3), $473-496$ (1998)

22. Abdelhedi, W, Chtioui, H: Prescribing mean curvature on $B^{n}$. Int. J. Math. 21(9), 1157-1187 (2010)

23. Li, YY: Prescribing scalar curvature on $S^{n}$ and related topics, part I. J. Differ. Equ. 120, 319-410 (1995)

24. Li, YY: Prescribing scalar curvature on $S^{n}$ and related topics, part II: existence and compactness. Commun. Pure Appl. Math. 49, 541-579 (1996)

25. Bahri, A: Critical Point at Infinity in Some Variational Problems. Pitman Res. Notes Math. Ser., vol. 182. Longman, Harlow (1989)

26. Bahri, A: An invariant for Yamabe-type flows with applications to scalar curvature problems in high dimension. A celebration of J.F. Nash Jr.. Duke Math. J. 81, 323-466 (1996)

27. Li, YY, Zhu, M: Uniqueness theorems through the method of moving spheres. Duke Math. J. 80, 383-417 (1995)

28. Bahri, A, Rabinowitz, P: Periodic orbits of Hamiltonian systems of three body type. Ann. Inst. Henri Poincaré, Anal. Non Linéaire 8, 561-649 (1991)

doi:10.1186/1029-242X-2013-405

Cite this article as: Al-Ghamdi et al.: On a geometric equation involving the Sobolev trace critical exponent. Journal of Inequalities and Applications 2013 2013:405. 